TRANSACTIONS OF THE

AMERICAN MATHEMATICAL SOCIETY

Volume 363, Number 3, March 2011, Pages 1509-1532

S 0002-9947(2010)05157-8

Article electronically published on October 8, 2010

\title{
GENERALIZED MANIFOLDS IN PRODUCTS OF CURVES
}

\author{
AKIRA KOYAMA, JÓZEF KRASINKIEWICZ, AND STANISŁAW SPIEŻ
}

\begin{abstract}
The intent of this article is to distinguish and study some $n$ dimensional compacta (such as weak n-manifolds) with respect to embeddability into products of $n$ curves. We show that if $X$ is a locally connected weak $n$-manifold lying in a product of $n$ curves, then $\operatorname{rank} H^{1}(X) \geq n$. If $\operatorname{rank} H^{1}(X)=n$, then $X$ is an $n$-torus. Moreover, if $\operatorname{rank} H^{1}(X)<2 n$, then $X$ can be presented as a product of an $m$-torus and a weak $(n-m)$-manifold, where $m \geq 2 n-\operatorname{rank} H^{1}(X)$. If $\operatorname{rank} H^{1}(X)<\infty$, then $X$ is a polyhedron. It follows that certain 2-dimensional compact contractible polyhedra are not embeddable in products of two curves. On the other hand, we show that any collapsible 2-dimensional polyhedron embeds in a product of two trees. We answer a question of Cauty proving that closed surfaces embeddable in a product of two curves embed in a product of two graphs. We construct a 2-dimensional polyhedron that embeds in a product of two curves but does not embed in a product of two graphs. This solves in the negative another problem of Cauty. We also construct a weak 2-manifold $X$ lying in a product of two graphs such that $H^{2}(X)=0$.
\end{abstract}

\section{Contents}

1. Introduction

2. Definitions and general properties of quasi-manifolds,

weak manifolds and ramified manifolds

5. Product structure of locally connected weak manifolds lying in products of curves

7. Embedding cones over polyhedra into products of trees 1526

8. A polyhedron that embeds in a product of two curves

but not in a product of two graphs - A solution of Cauty's problem

9. A ramified 2-manifold in a product of two graphs

with trivial 2nd cohomology

Appendix

Problems

Acknowledgments

References

Received by the editors April 3, 2008 and, in revised form, June 4, 2009, June 18, 2009, and July 2, 2009.

2010 Mathematics Subject Classification. Primary 54E45, 55N05, 57N35; Secondary 55M10, $57 \mathrm{Q} 05$.

Key words and phrases. Embeddings, locally connected compacta, weak manifolds, products of curves.

(C)2010 American Mathematical Society Reverts to public domain 28 years from publication 


\section{INTRODUCTION}

All spaces discussed in this paper are metrizable, and all mappings (also called maps) are continuous. By a compactum we mean a compact metric space, by a continuum we mean a connected compactum, and by a curve we mean a 1dimensional continuum.

By the celebrated Menger-Nöbeling theorem (cf. [H-W, p. 60] and [E]) every $n$ dimensional separable metric space embeds in the Euclidean $(2 n+1)$-space $\mathbb{R}^{2 n+1}$. In 1958 J. Nagata N1 discovered a remarkable modification of that theorem.

Theorem 1.1 (Nagata). Every $n$-dimensional metric space, $n \geq 2$, embeds in a topological product $X_{1} \times \cdots \times X_{n+1}$ of 1-dimensional metric spaces.

This theorem has drawn the attention of many topologists and originated a series of papers containing numerous improvements and related results (see e.g. $[\mathrm{Bw},[\mathrm{I}-\mathrm{M}],[\mathrm{L}, \mathrm{Mi}, \mathrm{Ol}],[\mathrm{Ste}, \mathrm{T}]$ ).

Nagata also wondered if in his theorem $n$ factors $X_{i}$ would suffice [N2, p. 163]. It appeared that it would not. Actually, in $1975 \mathrm{~K}$. Borsuk B3] proved the following elegant result.

Theorem 1.2 (Borsuk). The $n$-sphere $\mathbb{S}^{n}, n \geq 2$, does not embed in a product of $n$ curves.

In the present paper we shall see that some 2-dimensional contractible compact polyhedra also have the same property.

Thus every $n$-dimensional space embeds in a product of $n+1$-dimensional factors and $n$ factors do not suffice. On the other hand, in the literature one can find several results dealing with embeddability of $n$-dimensional compacta in products of $n$ 1-dimensional compacta. Let us cite some noteworthy examples.

Theorem 1.3 (Kuperberg). Any closed orientable 2-manifold different from $\mathbb{S}^{2}$ embeds in a product of two graphs (see $\underline{\mathrm{Ku}}$ ).

As usual, by a graph we mean a compact 1-dimensional polyhedron.

Theorem 1.4 (Cauty). A non-orientable, compact, connected, closed 2-manifold $M$ embeds in a product of two graphs if and only if the Euler characteristic $\chi(M) \leq-4$ (see [C]).

Theorem 1.5 (Pol). Any hereditarily indecomposable $n$-dimensional continuum embeds in a product of $n$ hereditarily indecomposable curves (see $[\mathrm{P}]$ ).

Theorem 1.6 (Gillman, Matveev, Rolfsen). Any compact, connected, n-dimensional PL manifold with boundary embeds in a product of $n$ finite-ods (see [G-M-R]).

An $m$-od is by definition the cone over a discrete space of $m$ points.

The above results of Borsuk, Kuperberg and Cauty and, on the other hand, some problems posed by Cauty motivated our research. Now we give a brief summary of major results of the present paper. First we establish some terminology.

An $n$-dimensional compactum $X, n \geq 1$, is said to be a weak $n$-manifold if for every point $x \in X$ there is an open neighborhood $W$ of $x$ such that no pair $(\bar{U}, \partial U)$, where $U \ni x$ is open and $\bar{U} \subset W$, extends to a pair $(P, Q)$ of absolute retracts with $\operatorname{dim} P=n$ and $\operatorname{dim} Q=n-1$. (For details see Section 2.) For instance, an $n$ dimensional compactum $X$ is a weak $n$-manifold if the exact Steenrod homology $H_{n}(X, X \backslash\{x\}) \neq 0$ for every $x \in X$ (see the Appendix). 
The symbol $H^{*}(\cdot)$ is used to denote the Čech cohomology with integer coefficients $\mathbb{Z}$. In the case where no confusion is likely to occur, we write $f^{*}$ instead of $H^{*}(f)$, where $f$ is a mapping.

Theorem 1.7 (cf. Theorem 3.1 and Theorem 2.9). Let $X$ be a locally connected weak $n$-manifold, $n \geq 2$, with $H^{1}(X)$ of finite rank. If $X$ embeds in a product of $n$ curves, then it embeds in a product of $n$ graphs, and therefore $X$ is a polyhedron.

It follows that if a closed $n$-manifold embeds in a product of $n$ curves, then it embeds in a product of $n$ graphs. In particular, for $n=2$, this gives a positive solution to a problem by Cauty in [C]. It appears that a similar problem explicitly stated in that paper - where the word "surface" is replaced by "2-dimensional polyhedron" - has a negative answer. Actually, we shall prove the following

Theorem 1.8 (cf. Theorem 8.2). There exists a compact 2-dimensional polyhedron that embeds in a product of two curves but does not embed in a product of two graphs.

We shall also prove a stronger version of the following

Theorem 1.9 (cf. Theorem 5.1). Let $X$ be a locally connected weak $n$-manifold in a product $X_{1} \times \cdots \times X_{n}$ of $n$ curves. Then $\operatorname{rank} H^{1}(X) \geq n$. If $\operatorname{rank} H^{1}(X)=n$, then $X=S_{1} \times \cdots \times S_{n}$, where each $S_{i}$ is a simple closed curve in $X_{i}$; therefore $X$ is an $n$-torus.

Corollary 1.10 (cf. Theorem 6.1). Let $X$ be a 2-dimensional compact connected polyhedron. If $X$ embeds in a product of two curves and $\operatorname{rank} H^{1}(X) \leq 2$, then $X$ collapses either on a point, a graph, or a torus. In particular, $X$ is collapsible if $H^{1}(X)=0$.

There are two well-known contractible but not collapsible polyhedra: the Borsuk example [B1] (which occurs in [Z] under the name "dunce hat") and the "Bing house"; cf. [R-S. Hence neither can be embedded in a product of two curves. We also have some other results on collapsible polyhedra.

Theorem 1.11 (Theorem 6.2). Any collapsible 2-dimensional polyhedron embeds in a product of two trees.

Combining Corollary 1.10 and Theorem 1.11 we obtain the following

Corollary 1.12. A compact acyclic 2-dimensional polyhedron embeds in a product of two graphs if and only if it is collapsible.

Theorem 1.13 (Theorem 7.1). The cone over an n-dimensional polyhedron embeds in a product of $n+1$ finite-ods.

Theorem 1.14 (Theorem 9.1). For every $n \geq 2$ there is a quasi-n-manifold $X$ lying in a product of $n$ graphs such that $H^{n}(X)=0$.

The definition of a quasi- $n$-manifold is given in Section 2. For $n \geq 4$ this result was first proved by the referee of the original version of this paper (for more information see Section 9).

This paper may be viewed as a sequel to [K-K-S 1]; its content is essentially a modified and extended version of a portion of [K-K-S]. 


\section{Definitions AND GENERAL PROPERTIES OF QUASI-MANIFOldS, WEAK MANIFOLDS AND RAMIFIED MANIFOLDS}

First we recall a classic notion. An $n$-dimensional compactum is said to be an $n$-manifold at a point $x$ if there is an open neighborhood $W$ of $x$ in $X$ that is an open $n$-disc. Obviously, $X$ is an $n$-manifold at every point $x \in X$ if and only if $X$ is a closed $n$-manifold. The property discussed in the next paragraph follows from the Borsuk Separation Theorem relating compact sets separating $\mathbb{S}^{n}, n \geq 1$, to essential mappings of those sets into $\mathbb{S}^{n-1}$ (cf. [E-S, p. 302]).

For an $n$-dimensional compactum $X$ which is an $n$-manifold at a point $x_{0} \in X$ there is an open neighborhood $W$ of $x_{0}$ in $X$ such that for every open set $U \ni x$ with $\bar{U} \subset W$ there is an essential map $\partial U \rightarrow \mathbb{S}^{n-1}$. (In fact, this holds for every neighborhood $W$ that is an open $n$-disc.)

This observation can be used to define a larger class of $n$-dimensional compacta. Namely, an $n$-dimensional compactum $X$ is said to be a quasi-n-manifold at a point $x \in X$ if there is an open neighborhood $W$ of $x$ in $X$ such that for every open set $U \ni x$ with $\bar{U} \subset W$ and $\operatorname{dim} \partial U \leq n-1$ there is an essential map $\partial U \rightarrow \mathbb{S}^{n-1}$. Notice that a curve $X$ is a quasi-1-manifold at $x \in X$ if and only if $x$ is not an endpoint of $X 11$ If $X$ is a quasi-n-manifold at $x$, then every neighborhood of $x$ is $n$-dimensional. A compactum $X$ is said to be a quasi-n-manifold if it is a quasi- $n$-manifold at every point. Any $n$-dimensional compactum that is a union of quasi- $n$-manifolds is a quasi- $n$-manifold.

There is an abundance of quasi- $n$-manifolds that are not $n$-manifolds. Both the "Bing house" and the "dunce hat" are examples of quasi-2-manifolds that are not 2-manifolds. These examples are widely known primarily for being 2-dimensional contractible and not collapsible compact polyhedra.

Now we shall show that for compact polyhedra the property of being a quasi- $n$ manifold can be expressed in terms of algebraic topology; see Theorem 2.2.

By the Hopf Classification Theorem (cf. [Sp, p. 431]) for any $n$-dimensional space $X$ the group $\widetilde{H}^{n}(X)$ is in one-to-one correspondence with the set of homotopy classes of maps $X \rightarrow \mathbb{S}^{n}$. Non-zero elements correspond to homotopy classes of essential maps.

Lemma 2.1. An $n$-dimensional compact polyhedron $X$ with a triangulation $K$ is a quasi-n-manifold at a vertex $v$ if and only if $\widetilde{H}^{n-1}(|\operatorname{lk}(v, K)|) \neq 0$, where $\operatorname{lk}(v, K)$ is the link of $v$ in $K{ }^{2}$

Proof. $\Rightarrow$ This implication immediately follows from the Hopf Classification Theorem because for every open neighborhood $W$ of $v$ in $X$ there is an open set $U \ni v$ with $\bar{U} \subset W$ such that $\partial U$ is homeomorphic to $|\operatorname{lk}(v, K)|$.

$\Leftarrow$ Let $S$ denote the star of $v$. Put $W=S \backslash|l k(v, K)|$ and suppose there is an open set $U$ with $\bar{U} \subset W$ and $\operatorname{dim} \partial U \leq n-1$ such that $\partial U$ admits no essential map into $\mathbb{S}^{n-1}$. Hence $\widetilde{H}^{n-1}(\partial U)=0$ and $S \backslash \partial U=U \cup(S \backslash \bar{U})$, where $U$ and $S \backslash \bar{U}$ are disjoint

\footnotetext{
${ }^{1}$ A point $x \in X$ is said to be of order $n$ if $n$ is the minimal number such that $x$ admits arbitrarily small open neighborhoods with boundaries containing at most $n$ points. Points of order 1 are called endpoints of the space.

${ }^{2}$ For a simplex $\sigma \in K$ the subcomplex $\operatorname{lk}(\sigma, K)$ and the corresponding point $\operatorname{set}|\operatorname{lk}(\sigma, K)|$ are both called links of $\sigma$ in $K$. A polyhedron $X=|K|$ need not be a quasi- $n$-manifold $(n \geq 2)$ if the link of every vertex of $K$ has non-trivial $(n-1)$-cohomology: the union of the boundaries of two disjoint $(n+1)$-simplices joined by a 1-simplex is a polyhedron of that type.
} 
and open in $S,|\operatorname{lk}(v, K)| \subset S \backslash \bar{U}$ and $v \in U$. Let $j: S \backslash\{v\} \rightarrow(S \backslash\{v\}, \partial U)$ denote the inclusion mapping. Then $j^{*}: \widetilde{H}^{n-1}(S \backslash\{v\}, \partial U) \rightarrow \widetilde{H}^{n-1}(S \backslash\{v\})$ is surjective. As $\widetilde{H}^{n-1}(S \backslash\{v\}) \simeq \widetilde{H}^{n-1}(|\operatorname{lk}(v, K)|)$, we shall get a contradiction proving that $j^{*}=0$. Since the homomorphism $h: \widetilde{H}^{n-1}(S \backslash\{v\}, S \backslash U) \oplus \widetilde{H}^{n-1}(S \backslash\{v\}, \bar{U} \backslash\{v\}) \rightarrow$ $\widetilde{H}^{n-1}(S \backslash\{v\}, \partial U)$ from the Mayer-Vietoris sequence is an isomorphism, it suffices to show that $j^{*} \circ h=0$. But $j^{*} \circ h$ factors through $\widetilde{H}^{n-1}(S \backslash\{v\},|\operatorname{lk}(v, K)|) \oplus$ $\widetilde{H}^{n-1}\left(S \backslash\{v\}, S^{\prime} \backslash\{v\}\right)$, where $S^{\prime} \subset U$ is the star of $v$ in a subdivision of $K$ and the latter direct sum is the trivial group.

Theorem 2.2. For any compact $n$-dimensional polyhedron $X=|K|$ and a point $x \in X$ the following are equivalent:

(i) $X$ is a quasi-n-manifold at $x$;

(ii) $\widetilde{H}^{n-1}(|\operatorname{lk}(x, L)|) \neq 0$ for every triangulation $L$ of $X$ with $x$ as a vertex;

(iii) either $\operatorname{dim} \sigma(x)=n$ or $\operatorname{dim} \sigma(x)<n$ and $\widetilde{H}^{n-\operatorname{dim} \sigma(x)-1}(|\operatorname{lk}(\sigma(x), K)|) \neq 0$, where $\sigma(x)$ is the carrier of $x$ in $K$;

(iv) $H^{n}(X, X \backslash\{x\}) \neq 0$;

(v) $\widetilde{H}^{n-1}(|\mathrm{k}(x, L)|) \neq 0$ for some triangulation $L$ of $X$ with $x$ as a vertex.

Proof. In the proof by $K^{\prime}$ we denote the simplicial subdivision of $K$ obtained by starring $K$ at $x$. Then $x$ is a vertex of $K^{\prime}$.

(i) $\Rightarrow$ (ii) Follows from Lemma 2.1.

(ii) $\Rightarrow$ (iii) We have $\left|\operatorname{lk}\left(x, K^{\prime}\right)\right|=\dot{\sigma}(x) *|\operatorname{lk}(\sigma(x), K)| 3$ On the other hand, $\dot{\sigma}(x) *$ $|\operatorname{lk}(\sigma(x), K)|$ is homeomorphic to $\Sigma^{\operatorname{dim} \sigma(x)}(|\operatorname{lk}(\sigma(x), K)|)$. Hence

$$
0 \neq \widetilde{H}^{n-1}\left(\left|\operatorname{lk}\left(x, K^{\prime}\right)\right|\right) \simeq \widetilde{H}^{n-\operatorname{dim} \sigma(x)-1}(|\operatorname{lk}(\sigma(x), K)|) .
$$

(iii) $\Rightarrow$ (iv) We have $\widetilde{H}^{n-1}\left(\left|\operatorname{lk}\left(x, K^{\prime}\right)\right|\right) \simeq \widetilde{H}^{n-\operatorname{dim} \sigma(x)-1}(|\mathrm{lk}(\sigma(x), K)|) \neq 0$. But $H^{n}(X, X \backslash\{x\}) \simeq H^{n}(S, S \backslash\{x\}) \simeq \widetilde{H}^{n-1}\left(\left|\operatorname{lk}\left(x, K^{\prime}\right)\right|\right)$, where $S$ is the star of $x$ in $K^{\prime}$. Hence $H^{n}(X, X \backslash\{x\}) \neq 0$.

(iv) $\Rightarrow$ (v) Using the same argument as above

$$
\widetilde{H}^{n-1}(|\operatorname{lk}(x, L)|) \simeq H^{n}(X, X \backslash\{x\}) \neq 0 .
$$

(v) $\Rightarrow$ (i) This follows from Lemma 2.1 .

Corollary 2.3. Let $X_{i}, i=1,2$, be compact polyhedra with $\operatorname{dim} X_{i}=n_{i}$. If $X_{1} \times X_{2}$ is a quasi- $\left(n_{1}+n_{2}\right)$-manifold at $\left(x_{1}, x_{2}\right) \in\left(X_{1} \times X_{2}\right)$, then $X_{i}$ is a quasi-n $n_{i}$-manifold at $x_{i}$. If $X_{1}$ is a quasi-n $n_{1}$-manifold at $x_{1} \in X_{1}$ and $X_{2}$ is a quasi- $n_{2}$-manifold at $x_{2} \in X_{2}$ with $n_{2} \leq 2$, then $X_{1} \times X_{2}$ is a quasi- $\left(n_{1}+n_{2}\right)$-manifold at $\left(x_{1}, x_{2}\right)$.

Proof. By the Künneth formula we have $H^{n_{1}+n_{2}}\left(X_{1} \times X_{2}, X_{1} \times X_{2} \backslash\left\{\left(x_{1}, x_{2}\right)\right\}\right) \simeq$ $H^{n_{1}}\left(X_{1}, X_{1} \backslash\left\{x_{1}\right\}\right) \otimes H^{n_{2}}\left(X_{2}, X_{2} \backslash\left\{x_{2}\right\}\right)$. Hence the conclusions follow from Theorem 2.2 and the fact that $H^{n_{2}}\left(X_{2}, X_{2} \backslash\left\{x_{2}\right\}\right)$ is free for $n_{2} \leq 2$.

A compact pair $(X, A)$ is said to extend to a pair $\left(X^{\prime}, A^{\prime}\right)$ if there is an embedding $h: X \rightarrow X^{\prime}$ such that $h(A) \subset A^{\prime}$.

A compact $n$-dimensional space $X$ is said to be a weak n-manifold at a point $x \in X$ if there is an open neighborhood $W$ of $x$ in $X$ such that no pair $(\bar{U}, \partial U)$, where $U \ni x$ is open and $\bar{U} \subset W$, extends to a compact pair $(P, Q)$ of absolute

${ }^{3}$ By definition, $A * \emptyset=A$. In particular, the suspension $\Sigma \emptyset=\mathbb{S}^{0} * \emptyset=\mathbb{S}^{0}$. 
retracts with $\operatorname{dim}(P, Q)=(n, n-1)$ (that is, $\operatorname{dim} P=n$ and $\operatorname{dim} Q=n-1)$ An $n$-dimensional compactum is said to be a weak $n$-manifold if it is a weak $n$-manifold at every point. A curve $X$ is a weak 1-manifold if $X$ is a local dendrite at none of its endpoints. Notice that

Lemma 2.4. If $X$ is a weak n-manifold at a point $x$, then every neighborhood of $x$ is n-dimensional.

Proof. This follows from the following observation: any compact pair $(Y, B)$ extends to a compact pair $\left(Y^{\prime}, B^{\prime}\right)$ of absolute retracts such that $\operatorname{dim}\left(Y^{\prime}, B^{\prime}\right)=(\operatorname{dim} Y+$ $1, \operatorname{dim} B+1)$ (see [Bo] ;f. [K], [K-O], [Kr2], and [Mil]).

Lemma 2.5. If $X$ is a quasi-n-manifold at a point $x$, then $X$ is a weak n-manifold at $x$.

Proof. Let $W$ be an open neighborhood of $x$ satisfying the definition of a quasi$n$-manifold at $x$, and let $U \ni x$ be open and such that $\bar{U} \subset W$. Suppose $(\bar{U}, \partial U)$ extends to a compact pair $(P, Q)$ of absolute retracts with $\operatorname{dim}(P, Q)=(n, n-1)$. Then $\operatorname{dim} \partial U \leq n-1$ as $\partial U \subset Q$. Hence there is an essential map $f: \partial U \rightarrow \mathbb{S}^{n-1}$. Then $f$ extends to a map $f^{*}: Q \rightarrow \mathbb{S}^{n-1}$ because $\operatorname{dim} Q=n-1$. Consequently, $f$ is homotopic to a constant because $Q$ is an absolute retract, a contradiction 5

In Proposition 2.7 we show that in the class of polyhedra the implication from Lemma 2.5 is reversible for $n \neq 3$, and with some restrictions also for $n=3$. First we need a lemma.

Lemma 2.6. Let $|L|$ be a compact $m$-dimensional polyhedron with $H^{m}(|L|)=0$, where $m \geq 1$. For $m=2$ assume in addition that $|L|$ is simply connected. Then $|L|$ extends to an m-dimensional contractible polyhedron.

Proof. Without loss of generality we can assume that $|L|$ is connected (otherwise we can connect the components by arcs preserving the simple connectedness).

For $m=1$ the polyhedron $|L|$ is a tree, so it is contractible.

For $m=2$ the polyhedron $|L|$ is either contractible or has the homotopy type of a bouquet of 2 -spheres, because it is simply connected. The latter case is excluded because $H^{2}(|L|)=0$.

Now consider the case $m \geq 3$. Let $\left|L^{\prime}\right|=|L| \cup c\left|L^{(m-2)}\right| 6$ Observe that $H^{m}\left(\left|L^{\prime}\right|\right) \simeq H^{m}(|L|)=0$. Hence by the universal coefficient theorem for cohomology [Sp, Chapter 5, Theorem 3, p. 243], $H_{m}\left(\left|L^{\prime}\right|\right)=0$ and $H_{m-1}\left(\left|L^{\prime}\right|\right)$ is free abelian. Note also that $\widetilde{H}_{k}\left(\left|L^{\prime}\right|\right)=0$ if $k<m-1$ and that $\left|L^{\prime}\right|$ is simply connected. Hence $\left|L^{\prime}\right|$ is a Moore space $M(G, m-1)$, where $G=H_{m-1}\left(\left|L^{\prime}\right|\right)$. By the uniqueness of Moore spaces (cf. [H] Section 4.2, Example 4.34, p. 368]) the polyhedron $\left|L^{\prime}\right|$ has the homotopy type of a PL bouquet $B$ of $(m-1)$-spheres. It follows that there is a PL homotopy equivalence $f: B \rightarrow\left|L^{\prime}\right|$. Hence the mapping cone $C_{f}$ of $f$ is a contractible $m$-dimensional polyhedron (cf. [Ma, Section 8.4, Theorem, p. 57] and $[$ Sp, Section 7.1, Theorem 9, p. 369]), so the conclusion follows.

\footnotetext{
${ }^{4}$ Equivalently, $X$ is not a weak $n$-manifold at $x$ if there exist arbitrarily small open sets $U \ni x$ such that $(\bar{U}, \partial U)$ extends to a compact pair $(P, Q)$ of absolute retracts with $\operatorname{dim}(P, Q)=(n, n-1)$.

${ }^{5}$ Notice that in the proof the extension of $\bar{U}$ to an $n$-dimensional absolute retract has not been used. It was enough to know that $\partial U$ extends to an $(n-1)$-dimensional absolute retract.

${ }^{6} \mathrm{By} K^{(m)}$ we denote the $m$ th skeleton of $K$; if $m<0$, then $K^{(m)}=\emptyset$ by definition. By $c X$ we denote the cone over $X$.
} 
Proposition 2.7. Let $P=|K|$ be an $n$-dimensional polyhedron with $n \geq 1$, and let $x \in P$ be any point. For $n=3$ assume in addition that $|l k(x, K)|$ is simply connected if $x$ is a vertex of $K$. Then $P$ is a quasi-n-manifold at $x$ if $P$ is a weak $n$-manifold at $x$.

Proof. For $n \leq 2$ the proof is straightforward. Now assume $n \geq 3$ and suppose $P$ is not a quasi- $n$-manifold at $x$. To end the proof, we have to show that $P$ is not a weak $n$-manifold at $x$.

Let $K^{\prime}$ denote the simplicial subdivision of $K$ obtained by starring $K$ at $x$. For each $\varepsilon>0$ there is a simplicial subdivision $L$ of $K^{\prime}$ such that mesh $L<\varepsilon$. Clearly, $x$ is a vertex of $L$. Therefore, it suffices to extend $|\operatorname{lk}(x, L)|$ to an $(n-1)$-dimensional contractible polyhedron $Q$ (by taking the cone $P=c Q$ containing the $\operatorname{star}|\operatorname{st}(x, L)|$ we get a compact pair $(P, Q)$ of absolute retracts with $\operatorname{dim}(P, Q)=(n, n-1)$ extending the pair $(|\operatorname{st}(x, L)|,|\operatorname{lk}(x, L)|))$.

By the supposition and Theorem 2.2(v) we infer that $H^{n-1}(|\operatorname{lk}(x, L)|)=0$. Consequently, if $n \geq 4$, then applying Lemma 2.6 to $|\operatorname{lk}(x, L)|$ we infer that $|\operatorname{lk}(x, L)|$ extends to an $(n-1)$-dimensional contractible polyhedron. Hence the proof is completed in this case.

Finally, assume $n=3$. Notice that

$$
|\operatorname{lk}(x, L)| \approx\left|\operatorname{lk}\left(x, K^{\prime}\right)\right| \approx \Sigma^{\operatorname{dim} \sigma(x)}|\operatorname{lk}(\sigma(x), K)| .
$$

If $\operatorname{dim} \sigma(x) \neq 1$, then $|\operatorname{lk}(x, L)|$ is simply connected and hence extends to a 2dimensional contractible polyhedron by Lemma 2.6.

If $\operatorname{dim} \sigma(x)=1$, then $H^{1}(|\operatorname{lk}(\sigma(x), K)|)=0$ by the supposition and Theorem 2.2 (iii). It follows that the components of the graph $|\operatorname{lk}(\sigma(x), K)|$ are contractible, and hence $|\operatorname{lk}(\sigma(x), K)|$ extends to a tree. Consequently, $\Sigma|\operatorname{lk}(\sigma(x), K)|$, hence also $|\mathrm{lk}(x, L)|$, extends to a 2-dimensional contractible polyhedron. This completes the proof.

A cell complex $\sqrt{7} K$ is called a ramified $n$-complex if $K$ consists of a finite collection of $n$-cells and their faces such that each $(n-1)$-cell is a face of at least two $n$-cells. (A ramified 0-complex consists of at least two points.) Any cell complex which can be presented as a finite union of ramified $n$-complexes is itself a ramified $n$-complex. The underlying polyhedron $X=|K|$ of a ramified $n$-complex $K$ is called a ramified $n$-manifold. The notion of a ramified $n$-manifold is independent of the choice of the cell complex: if $K$ is a ramified $n$-complex and $|L| \approx|K|$, where $L$ is a cell complex, then $L$ is a ramified $n$-complex as well (this follows from Theorem 2.2). Any polyhedron that is a quasi- $n$-manifold is a ramified $n$-manifold. For $n \leq 2$ the converse is also true 8

Our main object in this section is Theorem 2.9 below which describes a basic property of weak $n$-manifolds lying in $n$-dimensional polyhedra.

\footnotetext{
${ }^{7}$ The notion of cell complex and other related notions (such as that of cell, face, subcomplex, etc.) are understood in the sense of $[\mathrm{R}-\mathrm{S}]$. Notice that a cell complex is always a finite collection of cells in some $\mathbb{R}^{n}$. A cell is a convex polyhedron; the underlying space $|K|$ of a cell complex is a polyhedron. The relation of a face, $\sigma<\tau$, coincides with the inclusion $\sigma \subset \tau$. Any cell $\sigma$ of a cell complex $K$ is the underlying space of a subcomplex of $K$. The open cell $\%$ is by definition the geometric interior of the cell $\sigma$. It is homeomorphic to $\mathbb{R}^{n}$, where $n=\operatorname{dim} \sigma$; hence it is an open $n$-disc. The open cells are mutually disjoint and cover $|K|$.

${ }^{8}$ For $n=3$ it fails: the suspension of the Borsuk example [B1] is a counterexample. For $n \leq 2$ any ramified $n$-manifold is a quasi- $n$-manifold.
} 
Lemma 2.8. Let $X$ be a weak n-manifold and let $U$ be a non-void open subset of $X$. If $h: U \rightarrow \mathbb{R}^{n}$ is an embedding such that $h(U)$ is closed, then $h(U)=\mathbb{R}^{n}$.

Proof. Suppose $h(U)$ is a proper subset of $\mathbb{R}^{n}$. Then there is a segment $a b$ in $\mathbb{R}^{n}$ meeting $h(U)$ only at its endpoint $a$. Let $B_{r}, r>0$, denote the open $n$-ball in $\mathbb{R}^{n}$ with radius $r$ and center at $a$, and let $S_{r}$ be its boundary. Since $X$ is a weak $n$-manifold at $h^{-1}(a)$ there is an open neighborhood $W$ of $h^{-1}(a)$ in $X$ satisfying the definition. Then there is a positive number $r<d(a, b)$ such that $\bar{V} \subset h(U \cap W)$ for $V=B_{r} \cap h(U)$. Then $\partial_{h(U)} V \subset S_{r} \backslash a b$. Hence $\left(\bar{V}, \partial_{h(U)} V\right)$ extends to a pair of absolute retracts $\left(\overline{B_{r}}, D\right)$ with dimension $(n, n-1)$, where $D$ denotes a closed $(n-1)$ disc in $S_{r}$. Since $h^{-1}(V)$ is an open neighborhood of $h^{-1}(a)$ whose closure lies in $W$, and $h$ transforms $\left(\overline{h^{-1}(V)}, \partial h^{-1}(V)\right)$ homeomorphically onto $\left(\bar{V}, \partial_{h(U)} V\right)$, we get a contradiction.

Theorem 2.9. Let $X$ be a weak n-manifold. If $f: X \rightarrow|K|$ is an embedding, where $K$ is an $n$-dimensional cell complex, then $f(X)=|L|$, where $L$ is a ramified $n$-subcomplex of $K$.

Proof. First we prove that $f(X)$ is a union of $n$-cells of $K$. To this end, we have to show that each point $y \in f(X)$ is an element of an $n$-cell of $K$ that lies entirely in $f(X)$. There is an open neighborhood $V$ of $y$ in $f(X)$ such that, for each cell $\sigma \in K$, the condition $V \cap \sigma \neq \emptyset$ implies $y \in \sigma$. By Lemma 2.4 we have $\operatorname{dim} V=n$. Hence there is an $n$-cell $\sigma_{0} \in K$ such that $V \cap \stackrel{\circ}{\sigma}_{0} \neq \emptyset$. Therefore $y \in \sigma_{0}$. We shall show that $\sigma_{0} \subset f(X)$. As $f(X)$ is closed, it is enough to show that $\stackrel{\circ}{\sigma}_{0} \subset f(X)$. Notice that $f(X) \cap \stackrel{\circ}{\sigma}_{0}$ is non-void and open in $f(X)$. It follows that $U=f^{-1}\left(\stackrel{\circ}{\sigma}_{0}\right)$ is non-void and open in $X$ and that $f(U)=f(X) \cap \stackrel{\circ}{\sigma}_{0}$ is closed in $\stackrel{\circ}{\sigma}_{0}$. Since $\stackrel{\circ}{\sigma}_{0} \approx \mathbb{R}^{n}$, by Lemma 2.8 we infer that $f(X) \cap \stackrel{\circ}{\sigma}_{0}=\stackrel{\circ}{\sigma}_{0}$, which proves the inclusion. This proves the initial claim. Each cell of $K$ is the underlying space of a subcomplex of $K$, hence $f(X)=|L|$. It remains to show that each $(n-1)$-cell $\sigma \in L$ is a face of at least two $n$-cells of $L$. By the result we have just established $\sigma$ is a face of an $n$-cell $\sigma^{\prime} \in L$. If there is no other $n$-cell having $\sigma$ as its face, then one can easily verify that $f(X)$ is a quasi- $n$-manifold at no point $y \in \stackrel{\circ}{\sigma}$, a contradiction.

Lemma 2.10. Let $X$ be an $n$-dimensional compactum and let $Y$ be an $m$-manifold at a point $q$. If $X \times Y$ is a weak $(n+m)$-manifold at $(p, q)$, then $X$ is a weak $n$-manifold at $p$.

Proof. Suppose $X$ is not a weak $n$-manifold at $p$. Then there exist open sets $U \ni p$ in $X$ as small as we please such that $(\bar{U}, \partial U)$ extends to a compact pair $(P, Q)$ of absolute retracts with $\operatorname{dim}(P, Q)=(n, n-1)$. It follows from the hypothesis that there is a closed $m$-disc $D$ in $Y$ such that $q \in \stackrel{\circ}{D}$ and $D^{\circ}$ is open in $Y$. Then $V=U \times \stackrel{\circ}{D}$ is a neighborhood of $(p, q)$ in $X \times Y, \bar{V} \subset P \times D$ and $\partial V \subset Q^{\prime}$, where $Q^{\prime}=(P \times \partial D) \cup(Q \times D)$. Moreover, $P \times D$ is an $(m+n)$-dimensional absolute retract, and $\operatorname{dim} Q^{\prime}=n+m-1$. Notice that $Q^{\prime}$ is an absolute retract as well. In fact, it is a union of two ANRs that intersect along $Q \times \partial D$ which is also an ANR. Hence $Q^{\prime}$ is an ANR (cf. [B2, p. 90]). As $Q$ is a strong deformation retract of $P, Q \times D$ is a strong deformation retract of $Q^{\prime}$. Hence $Q^{\prime}$ is contractible, which implies that $Q^{\prime}$ is an absolute retract (cf. [B2, p. 122]). Thus $(\bar{V}, \partial V)$ extends to a compact pair of absolute retracts with dimension $(n+m, n+m-1)$. Since $D$ can be chosen as small as we please, $X \times Y$ is not a weak $(n+m)$-manifold at $(p, q)$, a contradiction. 


\section{FACtorization theorem}

First we prove Theorem 3.1 about factorization of embeddings of weak manifolds into products of curves through special embeddings into product of graphs containing no endpoint. Then we construct a closed surface in a product of two curves whose image under either projection is not a graph (Example 3.4).

Factorization Theorem 3.1. Let $X$ be a locally connected weak n-manifold with $H^{1}(X)$ of finite rank. If $f=\left(f_{1}, \cdots, f_{n}\right): X \rightarrow Y_{1} \times \cdots \times Y_{n}$ is an embedding in a product of $n$ curves, then there exist mappings $g=\left(g_{1}, \cdots, g_{n}\right): X \rightarrow P_{1} \times \cdots \times P_{n}$ and $h=h_{1} \times \cdots \times h_{n}: P_{1} \times \cdots \times P_{n} \rightarrow Y_{1} \times \cdots \times Y_{n}$ such that $f_{i}=h_{i} \circ g_{i}$ for each $i=1, \cdots, n$ (hence $f=h \circ g$ ), where $g_{i}: X \rightarrow P_{i}$ is a monotone surjection, $P_{i}$ is a graph with no endpoint, and $h_{i}: P_{i} \rightarrow Y_{i}$ is 0-dimensional. Thus $g$ is an embedding.

Note. The following observations follow from this theorem and its proof.

1. If $f_{i}: X \rightarrow Y_{i}$ is monotone, then $f_{i}(X)$ is a graph. In fact, in this case $f_{i}(X)=h_{i}\left(P_{i}\right)$ and $h_{i}: P_{i} \rightarrow Y_{i}$ is an embedding. (If $f_{i}$ is not monotone, then $f_{i}(X)$ need not be a graph; see Example 3.4.) If $X$ is a polyhedron (or a locally connected compactum with $H^{1}(X)$ of finite rank), then $g_{i}(X)$ is a 1-dimensional ANR.

2. Any locally connected weak 1-manifold with $H^{1}(X)$ of finite rank is a graph with no endpoint.

Proof. By the Whyburn factorization theorem [W, p. 141], there is a factorization $f_{i}=h_{i} \circ g_{i}$,

$$
X \stackrel{g_{i}}{\longrightarrow} P_{i} \stackrel{h_{i}}{\longrightarrow} Y_{i}
$$

where $g_{i}$ is a monotone surjection and $h_{i}$ is 0 -dimensional. Since $Y_{i}$ is 1-dimensional and $h_{i}$ is 0-dimensional, we infer that $\operatorname{dim} P_{i} \leq 1$ (by a theorem of Hurewicz $\mathrm{H}-\mathrm{W}$, p. 91]). Clearly, $g=\left(g_{1}, \cdots, g_{n}\right): X \rightarrow P_{1} \times \cdots \times P_{n}$ is an embedding. Since $\operatorname{dim} X=n$, it follows that $\operatorname{dim} P_{i}>0$ for each $i$. Therefore, $P_{i}$ is a 1-dimensional locally connected compactum because $g_{i}$ is a surjection. Since $g_{i}$ is a monotone surjection and $H^{1}(X)$ has finite rank, $P_{i}$ is actually a local dendrite (cf. [Kr1, Lemma 3.1]). Hence each point of $P_{i}$ has a closed neighborhood which is a dendrite. First we shall show that

$$
P_{i} \text { has no endpoint. }
$$

For suppose $P_{i}$ has an endpoint $z_{0}$. Since $g_{i}(X)=P_{i}$, there is a point $x_{0} \in X$ such that $g_{i}\left(x_{0}\right)=z_{0}$. Since $X$ is a weak $n$-manifold at $x_{0}$ there is an open neighborhood $W$ of $x_{0}$ in $X$ such that

(1) no pair $(\bar{V}, \partial V)$, where $V \ni x_{0}$ is open and $\bar{V} \subset W$, extends to a pair $(P, Q)$ of compact absolute retracts with $\operatorname{dim}(P, Q)=(n, n-1)$.

On the other hand, we shall show that there is an open neighborhood $U$ of $g\left(x_{0}\right)$ in $P_{1} \times \cdots \times P_{n}$ such that

(2) $\bar{U} \cap g(X \backslash W)=\emptyset$,

\footnotetext{
${ }^{9} \mathrm{By}$ a dendrite we mean a non-degenerate locally connected continuum containing no simple closed curve. A non-degenerate compactum is said to be a local dendrite (local tree, respectively) if every point has a closed neighborhood which is a dendrite (a tree, respectively). It is known that dendrites coincide with 1-dimensional compact ARs, and local dendrites with 1-dimensional compact ANRs (cf. Kur $)$.
} 
(3) $\operatorname{dim}(\bar{U}, \partial U)=(n, n-1)$,

(4) $(\bar{U}, \partial U)$ is a pair of compact absolute retracts.

To construct $U$ we assume, without loss of generality, that $i=1$. Then $g\left(x_{0}\right)=$ $\left(y_{1}, y_{2}, \cdots, y_{n}\right)$, where $y_{1}=z_{0}$. Note that $g(W)$ is a neighborhood of $g\left(x_{0}\right)$ in $g(X)$; hence any small enough $U$ satisfies (2). Since $z_{0}$ is an endpoint of $P_{1}$ and each $P_{j}$ is a local dendrite, there exist sets $U_{1}, \cdots, U_{n}$, as small as we please, such that each $U_{j}$ is an open and connected neighborhood of $y_{j}$ in $P_{j}$ with $\partial U_{j}$ finite, each $\overline{U_{j}}$ is a dendrite, and $\partial U_{1}$ is a one-point set. Then the set $U=U_{1} \times \cdots \times U_{n}$ has the desired properties. In fact, as $U_{j}$ 's are small, $U$ satisfies (2). Then note that

$$
\partial U=\bigcup_{j=1}^{n}\left(\overline{U_{1}} \times \cdots \times \overline{U_{j-1}} \times \partial U_{j} \times \overline{U_{j+1}} \times \cdots \times \overline{U_{n}}\right) .
$$

Hence (3) follows. To prove (4), first notice that the set $\bar{U}=\bar{U}_{1} \times \cdots \times \overline{U_{n}}$ is an absolute retract as a product of dendrites. Then notice that each product $\overline{U_{1}} \times \cdots \times \overline{U_{j-1}} \times \partial U_{j} \times \overline{U_{j+1}} \times \cdots \times \overline{U_{n}}$ is an ANR and that the intersection of any collection of such products is an ANR as well. It follows that $\partial U$ is an ANR (cf. [B2, p. 90]). Since $\partial U_{1}$ is a strong deformation retract of $U_{1}$, the product $\partial U_{1} \times \overline{U_{2}} \times \cdots \times \overline{U_{n}}$ is a strong deformation retract of $\partial U$. As the former set is an absolute retract, $\partial U$ is contractible. Hence $\partial U$ is an AR because it is a contractible ANR (cf. [B2, p. 122]).

Now consider a set $V^{\prime}=U \cap g(X) \ni g\left(x_{0}\right)$ open in $g(X)$ with $\overline{V^{\prime}} \subset g(W)$. Then $V=g^{-1}\left(V^{\prime}\right) \ni x_{0}$ is open in $X$ and $\bar{V} \subset W$. By (2)-(4) we infer that $\left(V^{\prime}, \partial V^{\prime}\right)$ extends to a compact pair $(\bar{U}, \partial U)$ of absolute retracts with $\operatorname{dim}(\bar{U}, \partial U)=(n, n-1)$. Since $g$ sends $(V, \partial V)$ homeomorphically onto $\left(V^{\prime}, \partial V^{\prime}\right)$, a similar extension holds for $(V, \partial V)$, contrary to $(1)$. This proves $(*)$.

Next we shall show that

$$
P_{i} \text { is a graph. }
$$

In order to prove $(* *)$ recall that $P_{i}$ is a local dendrite. Since $P_{i}$ has no endpoint, it contains a circle. (Otherwise, every non-degenerate component of $P_{i}$ is a dendrite; hence $P_{i}$ contains an endpoint because there is at least one such component.) Hence the union of all simple closed curves in $P_{i}$ is a graph. Then we can enlarge this graph to another graph $Q_{i}\left(\subset P_{i}\right)$ (by adding a finite collection of arcs irreducibly connecting those different components of the union which lie in the same components of $P_{i}$ ) so that for each component $C$ of $P_{i} \backslash Q_{i}$ we have

(5) $\bar{C}$ is a dendrite and $\partial C$ consists of a single point.

To complete the proof of (**) it suffices to show that $Q_{i}=P_{i}$. Suppose $P_{i} \backslash Q_{i} \neq$ $\emptyset$. Then consider a component $C$ of $P_{i} \backslash Q_{i}$. It is an open set in $P_{i}$. By (5), $\bar{C}$ is a dendrite and $\partial C$ is a one-point set. Hence there is a point $z_{0} \in C$ which is an endpoint of the dendrite $\bar{C}$. Since $C$ is open, $z_{0}$ is an endpoint of $P_{i}$ as well, contrary to $(*)$. This proves $(* *)$ and completes the proof of our theorem.

The mapping $g: X \rightarrow P_{1} \times \cdots \times P_{n}$ from Theorem 3.1 is an embedding. Hence by Theorem 2.9 we get the following

Corollary 3.2. Let $X$ be a locally connected weak n-manifold with $H^{1}(X)$ of finite rank. If $X$ embeds in a product of $n$ curves, then $X$ is a polyhedron that embeds in a product of $n$ graphs. 
Corollary 3.3. If a closed n-manifold embeds in a product of $n$ curves, then it is a polyhedron that embeds in a product of $n$ graphs.

Note. This provides an answer to a question posed (for surfaces) by R. Cauty [C].

Example 3.4. There exist a curve $X$ which is not a graph and a closed orientable surface $M$ in the product $X \times X$ such that both projections $p r_{i}: X \times X \rightarrow X$ map $M$ onto $X$. Moreover, $M$ is invariant under the canonical involution on $X \times X$ which interchanges the coordinates.

Proof. First we construct a closed orientable surface $N$ in the product $Y_{1} \times Y_{2}$ of two curves such that

(1) $Y_{1}$ is not a graph,

(2) the projections $q_{i}: Y_{1} \times Y_{2} \rightarrow Y_{i}$ map $N$ onto $Y_{i}$.

Define $Y_{1}$ to be the union $Y_{1}=\alpha_{0} \cup \alpha_{1} \cup \beta_{1} \cup \beta_{2}$ of four arcs with common endpoints $a, b$ such that $\alpha_{0} \cup \alpha_{1} \cup \beta_{1}$ ia a $\theta$-curve, $\beta_{2} \cap\left(\stackrel{\circ}{\alpha}_{0} \cup \stackrel{\circ}{\alpha}_{1}\right)=\emptyset$, and $\beta_{1} \cap \stackrel{\circ}{\beta}_{2}$ is a compact set with infinitely many components. Then $Y_{1}$ satisfies (1). Define $Y_{2}$ to be a graph given by the formula $Y_{2}=T_{0} \cup T_{1}$, where $T_{0}, T_{1}$ are two oriented circles whose intersection $T_{0} \cap T_{1}=L_{0} \cup L_{1}$, where $L_{0}, L_{1}$ are disjoint oriented arcs coherently oriented with each $T_{i}$. In such a case, each $T_{i}$ can be presented as the union of four arcs with disjoint interiors, $T_{i}=A_{i} \cup B_{i} \cup L_{0} \cup L_{1}$, such that $S_{1}=A_{0} \cup A_{1}$ and $S_{2}=B_{0} \cup B_{1}$ are disjoint circles. We define the surface $N$ in $Y_{1} \times Y_{2}$ by the formula

$$
N=\alpha_{0} \times T_{0} \cup \alpha_{1} \times T_{1} \cup \beta_{1} \times S_{1} \cup \beta_{2} \times S_{2} .
$$

One easily verifies that $N$ is an orientable surface satisfying (2).

To construct the promised example we proceed as follows. Choose a homeomorphism $h: \alpha_{0} \rightarrow A_{0}$. Then define $X$ to be the quotient space $X=\left(Y_{1} \sqcup Y_{2}\right) / x \sim h(x)$ for each $x \in \alpha_{0}$. By (1) we infer that $X$ is a curve but not a graph. Let $X_{i}=h_{i}\left(Y_{i}\right)$, where $h_{i}: Y_{i} \rightarrow X$ are canonical embeddings. Clearly, $X=X_{1} \cup X_{2}$ and $X_{1} \cap X_{2}=A$, where $A=h_{1}\left(\alpha_{0}\right)=h_{2}\left(A_{0}\right)$ is an arc. Let $t: Y_{1} \times Y_{2} \rightarrow Y_{2} \times Y_{1}$ denote the map given by $t(y, z)=(z, y)$. Then we define $M(\subset X \times X)$ as follows:

$$
M=\left[\left(h_{1} \times h_{2}\right)(N) \cup\left(h_{2} \times h_{1}\right)(t(N))\right] \backslash(\AA \times \AA) .
$$

One easily verifies that $M$ is invariant under canonical involution on $X \times X$. Since $\alpha_{0} \times A_{0} \subset N \subset Y_{1} \times Y_{2}, A_{0} \times \alpha_{0} \subset t(N) \subset Y_{2} \times Y_{1}$. Hence $A \times A=\left(h_{1} \times\right.$ $\left.h_{2}\right)\left(\alpha_{0} \times A_{0}\right) \subset\left(h_{1} \times h_{2}\right)(N) \subset\left(h_{1} \times h_{2}\right)\left(Y_{1} \times Y_{2}\right)=X_{1} \times X_{2}$. Likewise, $A \times A \subset$ $\left(h_{2} \times h_{1}\right)(t(N)) \subset X_{2} \times X_{1}$. Moreover, $\left(X_{1} \times X_{2}\right) \cap\left(X_{2} \times X_{1}\right)=A \times A$. Hence we have

$$
\left(h_{1} \times h_{2}\right)(N) \cap\left(h_{2} \times h_{1}\right)(t(N))=A \times A .
$$

Thus $M$ is the connected sum of orientable surfaces $\left(h_{1} \times h_{2}\right)(N)$ and $\left(h_{2} \times\right.$ $\left.h_{1}\right)(t(N))$; hence it is an orientable surface. Applying (2), we easily see that both projections $p r_{i}: X \times X \rightarrow X$ map $M$ onto $X$.

In connection with the above proof let us note the following fact.

Note. Let $M$ be any compactum lying in the product $P_{1} \times P_{2}$ of two graphs. If $A$ is an arc in $P_{1}$ with $p_{1}^{-1}(A)=A \times\left(S_{1} \cup \cdots \cup S_{k}\right), k \geq 2$, where $S_{1}, \ldots, S_{k}$ are disjoint circles in $P_{2}$, then $M$ can be embedded in the product $P_{1}^{\prime} \times P_{2}$ in such a way that $P_{1}^{\prime}$ is a curve and the image of $M$ under the projection $P_{1}^{\prime} \times P_{2} \rightarrow P_{1}^{\prime}$ is not a graph. (In fact, $P_{1}^{\prime}$ can be obtained from $P_{1}$ by adding an $\operatorname{arc} B$ with the same endpoints as that of $A$ in such a way that $A \cup B$ is not a graph.) 
The surface $M$ constructed in Example 3.4 meets the diagonal of $X \times X$. Below we present another example of a surface in the product $P \times P$, where $P$ is a graph, which is disjoint from the diagonal of the product and is invariant under the canonical involution on $P \times P$.

Example 3.5. There exist a graph $P$ and a closed orientable surface $M$ in $P \times P$ such that: $M$ is disjoint with the diagonal of $P \times P$, both projections $p r_{i}: P \times P \rightarrow P$ map $M$ onto $P$, and $M$ is invariant under the canonical involution on $P \times P$.

Proof. Fix any number $n \geq 4$. The graph $P$ is defined to be a subset of $\mathbb{S}^{1} \times I$ given by

$$
P=\left(\mathbb{S}^{1} \times\{0,1\}\right) \cup\left\{z_{0}, \cdots, z_{n-1}\right\} \times I,
$$

where $z_{j}=\exp \left(2 \pi i \frac{j}{n}\right), j=0, \cdots, n-1$. Then define arcs $A_{j} \times\{0\}, A_{j} \times\{1\}, I_{j}$ and circles $S_{j}$ in $P$ as follows:

$$
A_{j}=\left\{\exp (2 \pi i t): t \in\left[\frac{j}{n}, \frac{j+1}{n}\right]\right\}, \quad I_{j}=\left\{z_{j}\right\} \times I, \quad S_{j}=I_{j} \cup\left(A_{j} \times\{0,1\}\right) \cup I_{j+1} .
$$

(All indices in this construction are reduced modulo $n$.) Finally, define tori $T_{j}$ to be the subsets of $P \times P$ given by $T_{j}=S_{j} \times S_{j+2}$. Notice that the intersection $D_{j}=T_{i} \cap T_{j+1}=I_{j+1} \times I_{j+3}$ is a disc. Now we are ready to define the surface $M$; put $M=\left(T_{0} \cup \cdots \cup T_{n-1}\right) \backslash\left(\stackrel{\circ}{D}_{0} \cup \cdots \cup \stackrel{\circ}{D}_{n-1}\right)$. One easily verifies that $M$ has all the desired properties.

\section{RAMIFIED MANIFOLDS IN PRODUCTS OF GRAPHS - THE STRUCTURE LEMMA}

The main results of this section are the Structure Lemma 4.9 and the Embedding Lemma 4.10 that will play an important role in the proof of the principal result of the following section. To obtain these results we first establish several elementary properties of ramified $n$-manifolds lying in products of $n$ graphs. The properties will then be obtained by studying fibers of projections restricted to the ramified manifolds.

Let us consider cell complexes $K_{1}, \ldots, K_{n}, n \geq 2$. By $K_{1} \square \ldots \square K_{n}$ (or $\left.\square_{j=1}^{n} K_{j}\right)$ we denote the collection of cells given by

$$
K_{1} \square \cdots \square K_{n}=\left\{\sigma_{1} \times \cdots \times \sigma_{n}: \sigma \in K_{1}, \cdots, \sigma_{n} \in K_{n}\right\} .
$$

Then $\square_{j=1}^{n} K_{j}$ is a cell complex, called the product cell complex of $K_{1}, \cdots, K_{n}$, and the face relation is given by $\sigma_{1}^{\prime} \times \cdots \times \sigma_{n}^{\prime}<\sigma_{1} \times \cdots \times \sigma_{n}$ if and only if $\sigma_{1}^{\prime}<\sigma_{1}, \cdots, \sigma_{n}^{\prime}<\sigma_{n}$. The space $\left|K_{1}\right| \times \cdots \times\left|K_{n}\right|$ is the underlying polyhedron of this complex. Let

$$
\operatorname{pr}_{J}: \prod_{j=1}^{n}\left|K_{j}\right| \rightarrow \prod_{j \in J}\left|K_{j}\right|
$$

denote the projection, where $J$ is a non-void proper subset of $\{1, \cdots, n\}$. As above, the space $\prod_{j \in J}\left|K_{j}\right|$ is the underlying polyhedron of the cell complex $\square_{j \in J} K_{j}$. Notice that $p r_{J}$ determines a function (which we denote by the same symbol):

$$
p r_{J}: \square_{j=1}^{n} K_{j} \rightarrow \square_{j \in J} K_{j} .
$$

This function is cellular, that is, it sends cells to cells and faces of a cell $\sigma$ surjectively onto faces of $p r_{J}(\sigma)$. If $L^{\prime}$ is a subset of $\square_{j=1}^{n} K_{j}$, then $p r_{J}\left(L^{\prime}\right)=\left\{p r_{J}(\sigma): \sigma \in L^{\prime}\right\}$ is a subset of $\square_{j \in J} K_{j}$ and $p r_{J}\left(\left|L^{\prime}\right|\right)=\left|p r_{J}\left(L^{\prime}\right)\right|$. If $L^{\prime}$ is a subcomplex, then so is $p r_{J}\left(L^{\prime}\right)$. 
In the remaining part of this section we consider fixed graphs $P_{1}=\left|K_{1}\right|, \cdots, P_{n}=$ $\left|K_{n}\right|, n \geq 2$, where each $K_{i}$ is a finite 1-dimensional simplicial complex.

Lemma 4.1. If $L^{\prime}$ is a ramified n-subcomplex of $\square_{j=1}^{n} K_{j}$, then $\operatorname{pr}_{J}\left(L^{\prime}\right)$ is a ramified $n_{J}$-complex, where $n_{J}$ is the cardinality of $J$.

We shall also consider a fixed connected ramified $n$-manifold $M=|L|$, where $L$ is a connected ramified $n$-complex in $\square_{j=1}^{n} K_{j}$.

Let $J^{c}=\{1, \cdots, n\} \backslash J$. Each cell $\rho \in \square_{j=1}^{n} K_{j}$ has a unique presentation as a product of two cells: $\rho=\sigma \times \tau$, where $\sigma \in \square_{j \in J} K_{j}$ and $\tau \in \square_{j \in J^{c}} K_{j}$. Thus $\sigma=\operatorname{pr}_{J}(\rho)$ and $\tau=\operatorname{pr}_{J^{c}}(\rho)$. Then we obtain in succession the following three lemmas.

Lemma 4.2. For each cell $\sigma \times \tau \in L$ with $\sigma \in \square_{j \in J} K_{j}$ and $\tau \in \square_{j \in J^{c}} K_{j}$ there is an $n$-cell $\sigma^{\prime} \times \tau^{\prime} \in L$ with $\sigma^{\prime} \in \square_{j \in J} K_{j}$ and $\tau^{\prime} \in \square_{j \in J^{c}} K_{j}$ such that $\sigma<\sigma^{\prime}$ and $\tau<\tau^{\prime}$

For a cell $\tau \in \square_{j \in J^{c}} K_{j}$ let $L / \tau$ denote a subcomplex of $\operatorname{pr}_{J}(L)$ composed of all cells $\sigma \in \square_{j \in J} K_{j}$ such that $\sigma \times \tau \in L$. If $\tau<\tau^{\prime}$ and $\tau^{\prime} \in \operatorname{pr}_{J^{c}}(L)$, then $L / \tau \supset L / \tau^{\prime}$.

Lemma 4.3. If $\tau \in p r_{J^{c}}(L)$ is an $n_{J^{c}}$-cell, then $L / \tau$ is a ramified $n_{J}$-complex.

Lemma 4.4. We have the following equalities: $p r_{J}(L)=\bigcup\left\{L / \tau^{\prime}: \tau^{\prime}\right.$ is an $n_{J^{c}}$-cell of $\left.\operatorname{pr}_{J^{c}}(L)\right\}$;

$L / \tau=\bigcup\left\{L / \tau^{\prime}: \tau^{\prime} \in \operatorname{pr}_{J^{c}}(L)\right.$ is an $n_{J^{c}}$-cell with face $\left.\tau\right\}$ for any $\tau \in \operatorname{pr}_{J^{c}}(L)$. Thus, $L / \tau$ is a ramified $n_{J}$-complex.

A ramified $n$-complex $K$ is said to be irreducible if $K$ contains no proper ramified $n$-complex. In such a case $K$ is connected. If $|K|$ is a connected $n$-manifold, then $K$ is an irreducible ramified $n$-complex.

Lemma 4.5. Suppose each $L / v$, where $v$ is a vertex of $\operatorname{pr}_{J c}(L)$, is an irreducible ramified $n_{J}$-complex. Then $\operatorname{pr}_{J}(L)=L / \tau$ for any $\tau \in p r_{J^{c}}(L)$. Consequently, $L=p r_{J}(L) \square p r_{J^{c}}(L)$; so $M=p r_{J}(M) \times p r_{J^{c}}(M)$. In particular, the hypothesis is fulfilled if $\operatorname{pr}_{J}(L)$ is an irreducible ramified $n_{J}$-complex.

Proof. By Lemma 4.4 we have $\operatorname{pr}_{J}(L)=\bigcup\left\{L / \tau^{\prime}: \tau^{\prime}\right.$ is an $\left.n_{J^{c} \text {-cell of }} \operatorname{pr}_{J^{c}}(L)\right\}$. According to Lemma 4.3 each $L / \tau^{\prime}$ is a ramified $n_{J}$-complex. For a vertex $v \in \tau^{\prime}$ we have $L / v \supset L / \tau^{\prime}$, hence $L / v=L / \tau^{\prime}$ as $L / v$ is irreducible. Since each 1cell $v v^{\prime} \in \operatorname{pr}_{J^{c}}(L)$ is a face of an $n_{J^{c}}$-cell $\tau^{\prime} \in p r_{J^{c}}(L)$, we have $L / v=L / v^{\prime}$. Since $\operatorname{pr}_{J^{c}}(L)$ is connected, all the sets $L / v$ and $L / \tau^{\prime}$ coincide. If $v$ is a vertex of $\tau \in \operatorname{pr}_{J^{c}}(L)$ and $\tau<\tau^{\prime}$, then $L / v \supset L / \tau \supset L / \tau^{\prime}$, hence all $L / \tau$ coincide. Therefore, $p r_{J}(L)=L / \tau$ for every $\tau \in p r_{J^{c}}(L)$. The equality $L=p r_{J}(L) \square p r_{J^{c}}(L)$ follows.

To complete the proof, suppose $\operatorname{pr}_{J}(L)$ is an irreducible $n_{J}$-complex. Then $L / v=p r_{J}(L)$ because $L / v \subset p r_{J}(L)$ is a ramified $n_{J}$-manifold.

As a corollary we get the following

Lemma 4.6. For any $1 \leq j \leq n$ the image $\operatorname{pr}_{j}(M)$ is a circle if and only if $|L / v|$ is a circle for each vertex $v$ of $\operatorname{pr}_{\{j\}^{c}}(L)$.

By a circle we mean a simple closed curve. Put

$$
J_{M}=\left\{j \in\{1, \cdots, n\}: p r_{j}(M) \text { is a circle }\right\} .
$$

If $J_{M}$ is non-void, then $M$ is said to have projections onto circles; any $p r_{j}$ with $j \in J_{M}$ is said to project $M$ onto a circle. 
Lemma 4.7. If $J_{M}=\{1, \cdots, n\}$, then $M=p r_{1}(M) \times \cdots \times p r_{n}(M)$ is an $n$ torus. If some projections $\mathrm{pr}_{j}$ project $M$ onto a circle and some not, then $M=$ $p r_{J_{M}}(M) \times p r_{J_{M}^{c}}(M)$, where $\left.p r_{J_{M}}(M)=\prod_{j \in J_{M}} p r_{j}(M)\right)$ is an $n_{J_{M}}$-torus and $\operatorname{pr}_{J_{M}^{c}}(M)$ is a ramified $n_{J_{M}^{c}}$-manifold in $\prod_{j \in J_{M}^{c}} P_{j}$ having no projection onto a circle.

Proof. First notice that $\operatorname{pr}_{J_{M}}(M)$ coincides with the $n_{J_{M}}$-torus $\prod_{j \in J_{M}} p r_{j}(M)$. Indeed, $p r_{J_{M}}(M)$ is a ramified $n_{J_{M}}$-manifold by Lemma 4.1. Also, it is a subset of the torus $\prod_{j \in J_{M}} \operatorname{pr}_{j}(M)$ of the same dimension. Hence it coincides with the torus. The equality $M=p r_{J_{M}}(M) \times p r_{J_{M}^{c}}(M)$ follows from Lemma 4.5.

Lemma 4.8. Let $p_{i}: M \rightarrow P_{i}$ and $q_{i}: S_{i} \rightarrow M, i=1, \cdots, n$, be mappings such that

$$
H^{1}\left(p_{i} \circ q_{j}\right): H^{1}\left(P_{i}\right) \rightarrow H^{1}\left(S_{j}\right)
$$

is an epimorphism for each $i=j$ and the zero homomorphism for $i \neq j$. Then

$$
\varphi: H^{1}(M) \rightarrow H^{1}\left(S_{1}\right) \oplus \cdots \oplus H^{1}\left(S_{n}\right),
$$

given by $\varphi=\left(H^{1}\left(q_{1}\right), \cdots, H^{1}\left(q_{n}\right)\right)$, is an epimorphism. Consequently, $\operatorname{rank} H^{1}(M)$ $\geq \operatorname{rank} H^{1}\left(S_{1}\right)+\cdots+\operatorname{rank} H^{1}\left(S_{n}\right)$.

Proof. Let $\beta=\left(\beta_{1}, \cdots, \beta_{n}\right)$ be any element in $H^{1}\left(S_{1}\right) \oplus \cdots \oplus H^{1}\left(S_{n}\right)$. We need an $\alpha \in H^{1}(X)$ such that $\varphi(\alpha)=\beta$. By our assumption $\beta_{i}=H^{1}\left(p_{i} \circ q_{i}\right)\left(\alpha_{i}\right)$ for some $\alpha_{i} \in H^{1}\left(P_{i}\right)$ and each $i$. One easily sees that $\alpha=H^{1}\left(p_{1}\right)\left(\alpha_{1}\right)+\cdots+H^{1}\left(p_{n}\right)\left(\alpha_{n}\right)$ is the desired element.

Now we are ready to prove the main results of this section.

Structure Lemma 4.9. Let $M=|L|$, where $L$ is a connected ramified n-subcomplex of $\square_{j=1}^{n} K_{j}$. Then $\operatorname{rank} H^{1}(M) \geq n$. If $\operatorname{rank} H^{1}(M)=n+k$ and $k<n$, then $n_{J_{M}} \geq n-k$. If $J_{M}=\{1, \cdots, n\}$, then $M$ is the $n$-torus $\operatorname{pr}_{1}(M) \times \cdots \times p r_{n}(M)$. If $J_{M}$ is a proper non-void subset of $\{1, \cdots, n\}$, then $M=\operatorname{pr}_{J_{M}}(M) \times p r_{J_{M}^{c}}(M)$, where $\operatorname{pr}_{J_{M}}(M)=\prod_{j \in J_{M}} p r_{j}(M)$ is an $n_{J_{M}}$-torus and $p r_{J_{M}^{c}}(M)$ is a polyhedron that is a ramified $n_{J_{M}^{c}}$-manifold having no projection onto a circle.

Proof. Pick a vertex $v_{j} \in \operatorname{pr}_{\{j\}^{c}}(M)$, for each $j=1, \cdots, n$. Then $L / v_{j}$ is a ramified 1-manifold. To continue the proof we apply Lemma 4.8 as follows.

Let $q_{j}:\left|L / v_{j}\right| \rightarrow M$ be given by $x \rightarrow\left(x, v_{j}\right)$ for each $x \in\left|L / v_{j}\right|$. Then $\operatorname{pr}_{\{j\}^{c}}\left(q_{j}(x)\right)=v_{j}$ for each $x \in\left|L / v_{j}\right|$, and $\left(p r_{j} \mid M\right) \circ q_{j}:\left|L / v_{j}\right| \rightarrow P_{j}$ is the inclusion map. Since $P_{j}$ is a graph, then $H^{1}\left(\left(p r_{j} \mid M\right) \circ q_{j}\right)$ is an epimorphism. If $i \neq j$, then $H^{1}\left(\left(p r_{i} \mid M\right) \circ q_{j}\right)$ is the trivial homomorphism because $\left(p r_{i} \mid M\right) \circ q_{j}$ maps $\left|L / v_{j}\right|$ into a point. Thus (substituting $\left|L / v_{j}\right|$ in place of $S_{j}$ ) Lemma 4.8 implies

$$
\operatorname{rank} H^{1}(M) \geq \operatorname{rank} H^{1}\left(\left|L / v_{1}\right|\right)+\cdots+\operatorname{rank} H^{1}\left(\left|L / v_{n}\right|\right) .
$$

Notice that $\operatorname{rank} H^{1}\left(\left|L / v_{j}\right|\right) \geq 1$ because $\left|L / v_{j}\right|$ is a ramified 1-manifold; see Lemmas 4.3 and 4.4. Hence rank $H^{1}(M) \geq n$, which proves the first assertion.

Now suppose rank $H^{1}(M)=n+k$ and $k<n$. Choose the vertices $v_{j}$ so that $\operatorname{rank} H^{1}\left(\left|L / v_{j}\right|\right) \geq \operatorname{rank} H^{1}\left(\left|L / w_{j}\right|\right)$ for each vertex $w_{j} \in \operatorname{pr}_{\{j\}^{c}}(L)$. Let

$$
J_{0}=\left\{j \in\{1, \cdots, n\}: \operatorname{rank} H^{1}\left(\left|L / v_{j}\right|\right)=1\right\} .
$$

Since $\operatorname{rank} H^{1}(M)=n+k$, it follows from $(*)$ that $\operatorname{rank} H^{1}\left(\left|L / v_{j}\right|\right) \geq 2$ for at most $k$ indices $j$. Hence $J_{0}$ consists of at least $n-k$ indices. If $j \in J_{0}$, then $\operatorname{rank} H^{1}\left(\left|L / w_{j}\right|\right)=1$ for each vertex $w_{j} \in \operatorname{pr}_{\{j\}^{c}}(L)$. Hence $\left|L / w_{j}\right|$ is a circle. 
Therefore, by Lemma 4.5, $\operatorname{pr}_{j}(M)$ is a circle for each $j \in J_{0}$. It follows that $J_{0} \subset J_{M}$; hence $n_{J_{M}} \geq n-k$.

The remaining assertions directly follow from Lemma 4.7.

Embedding Lemma 4.10. Let $u: \prod_{j \in J} P_{j} \rightarrow Y$ and $v: \prod_{j \in J^{c}} P_{j} \rightarrow Y^{\prime}$ be mappings such that $u \times v: \prod_{i=1}^{n} P_{j} \rightarrow Y \times Y^{\prime}$ is injective on $M$. Then we have:

(i) If any two sets of the form $\operatorname{pr}_{J^{c}}\left(M \cap p r_{J}^{-1}(x)\right)$ with $x \in \operatorname{pr}_{J}(M)$ intersect, then $u$ is injective on $\operatorname{pr}_{J}(M)$.

(ii) If $J=\{j\}$, then $\operatorname{pr}_{j}(M)$ is a circle if and only if $\operatorname{pr}_{Y}((u \times v)(M))$ is a circle.

Proof. (i) Suppose $u(x)=u\left(x^{\prime}\right)$ for some $x, x^{\prime} \in p r_{J}(M)$. It suffices to show that $x=x^{\prime}$. By the hypothesis there is $y \in p r_{J^{c}}\left(M \cap p r_{J}^{-1}(x)\right) \cap p r_{J^{c}}\left(M \cap p r_{J}^{-1}\left(x^{\prime}\right)\right)$. Hence $(x, y),\left(x^{\prime}, y\right) \in M$ and $(u \times v)(x, y)=(u \times v)\left(x^{\prime}, y\right)$. Since $u \times v$ is injective on $M$ the conclusion follows.

(ii) $\Rightarrow$ Assume $p r_{j}(M)$ is a circle. According to Lemma $4.5 M=p r_{j}(M) \times$ $p r_{\{j\}^{c}}(M)$. Since $u \times v$ is injective on $M$ it follows that $u$ is injective on $p r_{j}(M)$. Thus $\operatorname{pr}_{Y}((u \times v)(M))=u\left(p r_{j}(M)\right)$ is a circle.

$\Leftarrow$ Assume $\operatorname{pr}_{Y}((u \times v)(M))$ is a circle. We have to show that so is $p r_{j}(M)$. By Lemma 4.5 it suffices to show that $|L / w|$ is a circle for each vertex $w \in p r_{\{j\}^{c}}(M)$. To this end, observe that for each vertex $w \in \operatorname{pr}_{\{j\}^{c}}(M)$ the set $|L / w|$ is a ramified 1-manifold, see Lemmas 4.4 and 4.3. Since $u \times v$ is injective on $|L / w| \times\{w\}$ it follows that $u$ is injective on $|L / w|$. Thus the ramified 1-manifold $u(|L / w|)$ lying in a circle $\operatorname{pr}_{Y}((u \times v)(M))$ is a circle as well. Hence $|L / w|$ is a circle.

In Section 8 we shall need the following very special case of the Structure Lemma 4.9. For the convenience of the reader we present this case with a substantially shorter proof (that is an extract from the general proof).

Lemma 4.11. If $M \subset P_{1} \times P_{2}$ is a topological torus then $M=S_{1} \times S_{2}$ for some circles $S_{i} \subset P_{i}$.

Proof. As before, $M=|L|$, where $L$ is a subcomplex of $K_{1} \square K_{2}$. Then, as in the proof of Lemma 4.9, it follows that

$$
2=\operatorname{rank} H^{1}(M) \geq \operatorname{rank} H^{1}\left(\left|L / v_{1}\right|\right)+\operatorname{rank} H^{1}\left(\left|L / v_{2}\right|\right),
$$

where $v_{1}$ is a vertex of $\operatorname{pr}_{2}(L)$ and $v_{2}$ is a vertex of $\operatorname{pr}_{1}(L)$. Since $\left|L / v_{i}\right|$ is a ramified 1-manifold, one gets $\operatorname{rank} H^{1}\left(\left|L / v_{i}\right|\right) \geq 1$; hence $\operatorname{rank} H^{1}\left(\left|L / v_{i}\right|\right)=1$. Therefore $\left|L / v_{i}\right|$ is a circle. By Lemma 4.6 we infer that $\operatorname{pr}_{i}(M)$ is a circle. Hence $M=p r_{1}(M) \times p r_{2}(M)$.

\section{Product structure of locally connected Weak Manifolds LYING IN PRODUCTS OF CURVES}

In this section we prove Theorem 5.1 on algebraic and splitting properties of connected and locally connected weak $n$-manifolds lying in products of $n$ curves. In the preceding section we have established notation related to a subset $M$ lying in a product of graphs $P_{1} \times \cdots \times P_{n}$. Here we use similar notation and terminology related to a subset $X$ of a product of curves $Y_{1} \times \cdots \times Y_{n}$. For instance, put

$$
J_{X}=\left\{j \in\{1, \cdots, n\}: p r_{j}(X) \text { is a circle }\right\} .
$$


Structure Theorem 5.1. Let $X$ be a connected and locally connected weak $n$ manifold with $H^{1}(X)$ of finite rank in a product $Y_{1} \times \cdots \times Y_{n}$ of $n$ curves, $n \geq 1$. Then $X$ is a polyhedron and the following conditions are fulfilled:

(1) $\operatorname{rank} H^{1}(X) \geq n$.

(2) If $\operatorname{rank} H^{1}(X)=n+k$, where $k<n$, then $J_{X}$ contains at least $n-k$ elements. In particular, if rank $H^{1}(X)=n$, then $J_{X}=\{1, \cdots, n\}$.

(3) If $J_{X}=\{1, \cdots, n\}$, then $X=p r_{1}(X) \times \cdots \times p r_{n}(X)$ is an $n$-torus.

(4) If $J_{X}$ is a proper non-void subset of $\{1, \cdots, n\}$, then $X=\left(\prod_{j \in J_{X}} \operatorname{pr}_{j}(X)\right) \times$ $\operatorname{pr}_{J_{X}^{c}}(X)$, where the first factor is an $n_{J_{X}}$-torus and $p r_{J_{X}^{c}}(X)$ is a polyhedron that is a weak $n_{J_{X}^{c}}$-manifold in $\prod_{j \in J_{X}^{c}} Y_{j}$ having no projection onto a circle.

If, in addition, $X$ is a quasi-n-manifold, then $\operatorname{pr}_{J_{X}^{c}}(X)$ is a quasi- $n_{J_{M}^{c}}$-manifold.

Proof. Let $f=\left(f_{1}, \cdots, f_{n}\right): X \rightarrow Y_{1} \times \cdots \times Y_{n}$ denote the inclusion, i.e. $f_{j}=p r_{j} \mid X$ for each $j$. By Theorem 3.1, there exist mappings $g=\left(g_{1}, \cdots, g_{n}\right): X \rightarrow P_{1} \times$ $\cdots \times P_{n}$ and $h=h_{1} \times \cdots \times h_{n}: P_{1} \times \cdots \times P_{n} \rightarrow Y_{1} \times \cdots \times Y_{n}$ such that $f=h \circ g$ (hence $f_{j}=h_{j} \circ g_{j}$ for each $j$ ), where each $g_{j}: X \rightarrow P_{j}=\left|K_{j}\right|$ is a surjection and $K_{j}$ is a simplicial 1-complex. Then $g$ is an embedding, hence $M=g(X)$ is a connected weak $n$-manifold in $P_{1} \times \cdots \times P_{n}$. By Theorem 2.9, $M=|L|$, where $L$ is a connected ramified $n$-subcomplex of $K_{1} \square \cdots \square K_{n}$. Hence $X \approx|L|$ is a polyhedron. By the Structure Lemma 4.9, $\operatorname{rank} H^{1}(X)=\operatorname{rank} H^{1}(M) \geq n$, which proves (1). By the Embedding Lemma 4.10(ii) we obtain $J_{X}=J_{M}$. In fact, $h$ as a product of mappings $u=h_{j^{\prime}}: P_{j^{\prime}} \rightarrow Y_{j^{\prime}}$ and $v=\prod_{j \in\left\{j^{\prime}\right\}^{c}} h_{j}: \prod_{j \in\left\{j^{\prime}\right\}^{c}} P_{j} \rightarrow$ $\prod_{j \in\left\{j^{\prime}\right\}^{c}} Y_{j}$ is injective on $M$ and $X=h(M)$. So, the hypotheses of the lemma are fulfilled. Hence the equality $J_{X}=J_{M}$ follows. It is easy to verify (2). In fact, suppose $\operatorname{rank} H^{1}(X)=n+k$, where $k<n$. Then, by the Structure Lemma, $J_{X}\left(=J_{M}\right)$ contains at least $n-k$ elements, which ends the verification. Condition (3) follows as in the Structure Lemma. Indeed, suppose $J_{X}=\{1, \cdots, n\}$. Then $X \subset p r_{1}(X) \times \cdots \times p r_{n}(X)$, hence $X=p r_{1}(X) \times \cdots \times p r_{n}(X)$ because $X$ is a weak $n$-manifold; cf. Theorem 2.9. Finally we shall prove (4). So, suppose $J_{X}$ is a proper non-void subset of $\{1, \cdots, n\}$. By the Structure Lemma we have $M=p r_{J_{M}}(M) \times p r_{J_{M}^{c}}(M)$, where $\operatorname{pr}_{J_{M}}(M)=\prod_{j \in J_{M}} p r_{j}(M)$ is an $n_{J_{M}}$-torus. Moreover, $\operatorname{pr}_{J_{M}^{c}}(M)$ is a polyhedron that by Lemma 2.10 is a weak $n_{J_{M}^{c}}$-manifold. Since $h=h_{1} \times \cdots \times h_{n}$ and $h \mid M$ is an embedding, the mapping $\left(\prod_{j \in J_{M}} h_{j}\right) \mid p r_{J_{M}}(M)$ is an embedding. Hence each $h_{j} \mid p r_{j}(M), j \in J_{M}$, is an embedding whose image equals $p r_{j}(X)$. Also, $\left(\prod_{j \in J_{M}^{c}} h_{j}\right) \mid p r_{J_{M}^{c}}(M)$ is an embedding whose image equals $p r_{J_{X}^{c}}(X)$. Therefore, $X=\left(\prod_{j \in J_{X}} p r_{j}(X)\right) \times p r_{J_{X}^{c}}(X)$, where each $p r_{j}(X), j \in J_{X}$, is a circle. Since for no $j \in J_{X}^{c}$ the set $p r_{j}(X)$ is a circle, the set $\operatorname{pr}_{J_{X}^{c}}(X)$ has no projection onto a circle.

The last assertion follows from Corollary 2.3.

Corollary 5.2. Let $X$ be a connected and locally connected weak n-manifold with $H^{1}(X)$ of finite rank in a product $Y_{1} \times \cdots \times Y_{n}$ of $n$ curves, $n \geq 1$. If no $p_{j}(X)$ is a circle, then $\operatorname{rank} H^{1}(X) \geq 2 n$.

Applying the Künneth formula we infer the following

Corollary 5.3. No product $\mathbb{T}^{n-k} \times M$, where $2 \leq k \leq n$, of an $(n-k)$-torus and a closed connected $k$-manifold $M$ with $H^{1}(M)=0$ embeds in a product of $n$ curves.

Remark. This corollary implies the Borsuk theorem [B3]. 
Corollary 5.4. Both the "Bing house" and the "dunce hat" are 2-dimensional compact contractible polyhedra not embeddable in a product of two curves.

Note. This corollary shows that the number of factors in the Nagata Embedding Theorem cannot be reduced to $n$, even for contractible $n$-dimensional polyhedra.

Corollary 5.5. Let $X$ be a closed connected $n$-manifold in a product $Y_{1} \times \cdots \times Y_{n}$ of $n$ curves, where $n \geq 2$. If rank $H^{1}(X) \leq n+1$, then $X=S_{1} \times \cdots \times S_{n}$, where each $S_{j}$ is a circle in $Y_{j}$.

Proof. By Theorem 5.1 there is a set $J$ composed of all elements of $\{1, \cdots, n\}$ but one $i$, such that

$$
X=\left(\prod_{j \in J} S_{j}\right) \times X^{\prime}
$$

where each $S_{j}$ is a circle in $Y_{j}$ and $X^{\prime} \subset Y_{i}$ is a polyhedron that is a quasi-1manifold. Since rank $H^{1}\left(X^{\prime}\right) \leq 2$, it follows that $X^{\prime}$ is a graph with no endpoint. As $X$ is an $n$-manifold, by $(*)$ it follows that $X^{\prime}$ contains no triod. Hence $X^{\prime}$ is a circle because it is connected.

\section{Polyhedra in Products of CURVES AND COllapsing}

The first result of this section follows from the Structure Theorem 5.1. It asserts, in particular, that any compact contractible 2-polyhedron lying in a product of two curves is collapsible. We shall also prove that any compact collapsible 2-dimensional polyhedron (in particular, the cone over a graph) embeds in a product of two trees.

Theorem 6.1. Let $P$ be a 2-dimensional compact connected polyhedron in a product of two curves. Then we have:

(i) If $H^{1}(P)=0$, then $P$ is collapsible.

(ii) If rank $H^{1}(P)=1$, then $P$ collapses on a circle.

(iii) If rank $H^{1}(P)=2$, then $P$ collapses on a torus or a ramified 1-manifold.

Remark. This result also implies the Borsuk theorem for $n=2[\mathrm{~B} 3]$.

Proof. The polyhedron $P$ collapses on a subpolyhedron $Q$ that does not collapse on any of its proper subpolyhedron. First observe the following:

(1) $Q$ is connected and $H^{1}(Q)=H^{1}(P)$.

(2) If $Q$ is 1 -dimensional, then it is a quasi-1-manifold ( so $\left.\operatorname{rank} H^{1}(Q) \geq 1\right)$.

(3) If $Q$ is 2-dimensional and $\operatorname{rank} H^{1}(P) \leq 2$, then $Q$ is a torus.

Only (3) needs a proof. So, let $X$ denote the union of all 2-simplices of $Q$. Then $X$ is a ramified 2-manifold, hence a quasi-2-manifold. Since $Q \backslash X$ is at most 1dimensional, the homomorphism $H^{1}(Q) \rightarrow H^{1}(X)$ induced by the inclusion $X \rightarrow Q$ is an epimorphism. Hence, by the assumption, $\operatorname{rank} H^{1}(X) \leq 2$. Now, the Structure Theorem 5.1(i) implies that $X$ is connected and $\operatorname{rank} H^{1}(X)=2$. Hence $X$ is a torus, by the Structure Theorem 5.1(2),(3). It remains to show that $X=Q$. Suppose, to the contrary, that $X$ is a proper subset of $Q$. Then there is a circle $S \subset Q$ not contained in $X$. Consequently, we get $\operatorname{rank} H^{1}(Q) \geq \operatorname{rank} H^{1}(X \cup S) \geq$ 3 , a contradiction.

The assertions (i)-(iii) now follow from properties (1)-(3). In fact, if $H^{1}(P)=0$, then $Q$ is a point, if $\operatorname{rank} H^{1}(P)=1$, then $Q$ is a circle, and if $\operatorname{rank} H^{1}(P)=2$, then $Q$ is either a torus or a ramified 1-manifold. 
Theorem 6.2. Let $K$ be a 2-dimensional simplicial complex. If $K$ collapses on a point, then $|K|$ embeds in a product of two trees.

Proof. By induction on the number of simplices of $K$ it suffices to prove the following assertion.

Let $K \searrow K^{\prime}$ be an elementary simplicial collapse of a simplicial complex $K$ on a subcomplex $K^{\prime}$, and let $f^{\prime}:\left|K^{\prime}\right| \rightarrow\left|K_{1}^{\prime}\right| \times\left|K_{2}^{\prime}\right|$ be an embedding that sends every simplex of $K^{\prime}$ onto a union of cells of $K_{1}^{\prime} \square K_{2}^{\prime}$. Then $K_{i}^{\prime}$ extends to a simplicial complex $K_{i}$ by adding 1-simplices, each of which meets $K_{i}^{\prime}$ at a single vertex, and $f^{\prime}$ extends to an embedding $|K| \rightarrow\left|K_{1}\right| \times\left|K_{2}\right|$ that sends every simplex of $K$ onto a union of cells of $K_{1} \square K_{2}$.

First suppose that $K \backslash K^{\prime}=\left\{\triangle^{2}, \triangle^{1}\right\}$, where $\triangle^{i}$ is an $i$-simplex, and let $\left(v_{1}^{1}, v_{1}^{2}\right)$, $\cdots,\left(v_{n}^{1}, v_{n}^{2}\right)$ be the vertices of the $\operatorname{arc} f^{\prime}\left(\triangle \cap\left|K^{\prime}\right|\right)$. Then $f^{\prime}$ extends to an embedding of $|K|$ in $\left|K_{1}\right| \times\left|K_{2}\right|$, where $K_{i}=K_{i}^{\prime} \bigcup_{v_{j}^{i}=(0, j)} I \times\{1, \ldots, n\}$, sending each $\triangle^{1}$ and $\triangle^{2}$ onto a union of cells. The case $K \backslash K^{\prime}=\left\{\triangle^{1}, \triangle^{0}\right\}$ is trivial.

\section{EMBEDding CONES OVER POLYHEDRA INTO PRODUCTS OF TREES}

Here we prove the following property of polyhedra.

Theorem 7.1. The cone over an n-dimensional polyhedron can be embedded in a product of $n+1$ copies of a finite-od.

Proof. We prove this by induction on $n$. For $n=0$ it is obvious. Then successively applying the following lemma (each time with $m=n-1$ ), we complete the proof.

Lemma 7.2. Let $K^{(m)}$ be the $m$-skeleton of a simplicial complex $K$, where $0 \leq$ $m \leq \operatorname{dim} K-1$. Let $L$ be the dual to $K^{(m)}$ in $K$. Then the cone over $|K|$ embeds in the product of cones over $\left|K^{(m)}\right|$ and $|L|$.

Proof. By definition $L$ is the collection of all simplices of the barycentric subdivision of $K$ which are disjoint with $\left|K^{(m)}\right|$. Notice that $|K|$ is pl isomorphic to a subpolyhedron of the join $\left|K^{(m)}\right| *|L|$. On the other hand, the cone over the join $\left|K^{(m)}\right| *|L|$ is homeomorphic to the product of cones over $\left|K^{(m)}\right|$ and $|L|$. This follows from the following formula for the link of a vertex in a product of two cones: $\operatorname{lk}\left(\left(a^{\prime}, a^{\prime \prime}\right), a^{\prime} P^{\prime} \times a^{\prime \prime} P^{\prime \prime}\right) \approx P^{\prime} * P^{\prime \prime}$; see [R-S, p. 24, Ex. (3)].

Notice that there is no natural extension of Theorem 7.1 to a more general case of compacta: the cone over the Menger curve does not embed in a product of two cones over 0-dimensional compacta.

\section{A POLYHEDRON THAT EMBEDS IN A PRODUCT OF TWO CURVES} BUT NOT IN A PRODUCT OF TWO GRAPHS-A SOLUTION OF CAUTY'S PROBLEM

The main result of this section is Theorem 8.2, which asserts that there exists a 2-dimensional polyhedron which embeds in a product of two curves but does not embed in a product of two graphs. This provides a negative solution to a problem posed by Cauty [C]. The construction strongly depends on Lemma 4.11: Any topological torus in a product of two graphs is a product of two circles lying in corresponding coordinate curves. 
Let us recall that by a $\theta$-curve we mean a union of three arcs having common endpoints and otherwise mutually disjoint. The arcs are called edges, and the endpoints are called vertices of the curve.

In all subsequent discussions any product of two 1-dimensional regular $C W$-complexes will be considered with its product cell structure 10 Any arc will be considered with its natural cell structure.

Lemma 8.1. Let $P$ be a $\theta$-curve and let $h: P \times P \rightarrow Y_{1} \times Y_{2}$ be an embedding in a product of two graphs. Then for any edge $\sigma$ in $P$ there exist arcs $\sigma_{i} \subset Y_{i}, i=1,2$, such that $h(\sigma \times \sigma)=\sigma_{1} \times \sigma_{2}$ and $h \mid \sigma \times \sigma: \sigma \square \sigma \rightarrow \sigma_{1} \square \sigma_{2}$.

Proof. It suffices to show that $h(\sigma \times \sigma)$ can be presented as a product of two arcs (lying in the corresponding graphs) such that $h$ sends each pair $\partial \sigma \times \sigma$ and $\sigma \times \partial \sigma$ of opposite 1-cells in $\sigma \times \sigma$ onto a corresponding pair of opposite 1-faces in the product. First we prove the following:

(1) If $S$ is a circle in $P$, then $h(\sigma \times S)$ is a product of a subset of $Y_{1}$ and a subset of $Y_{2}$ such that one of the subsets is an arc and the other is a circle.

Let $S_{1}$ and $S_{2}$ be circles in $P$ such that $\sigma=S_{1} \cap S_{2}$. Then $h\left(S_{i} \times S\right)=C_{i} \times D_{i}$, where $C_{i} \subset Y_{1}$ and $D_{i} \subset Y_{2}$ are circles. Observe that

$$
h(\sigma \times S)=h\left(S_{1} \times S\right) \cap h\left(S_{2} \times S\right)=\left(C_{1} \cap C_{2}\right) \times\left(D_{1} \cap D_{2}\right) .
$$

Notice that each of the sets $C_{1} \cap C_{2}$ and $D_{1} \cap D_{2}$ is a closed connected subset of a circle. Hence one of them is an arc and the other is a circle, because $h(\sigma \times S)$ is a topological cylinder. This proves (1).

Now, fix $S$ to be a circle in $P$ containing $\sigma$. It follows from (1) that

(2) $h(\sigma \times S)=A_{1} \times B_{1}, h(S \times \sigma)=A_{2} \times B_{2}$, where $A_{i} \subset Y_{1}, B_{i} \subset Y_{2}$, and each set $\left\{A_{i}, B_{i}\right\}$ consists of an arc and a circle.

Without loss of generality we can assume that $A_{1}$ is an arc and $B_{1}$ is a circle. Since $\sigma \times \sigma=(\sigma \times S) \cap(S \times \sigma)$ we get

(3) $h(\sigma \times \sigma)=\left(A_{1} \cap A_{2}\right) \times\left(B_{1} \cap B_{2}\right)$.

Since $h(\sigma \times \sigma)$ is a 2-disc, both $A_{1} \cap A_{2}$ and $B_{1} \cap B_{2}$ are arcs; put $\sigma_{1}=A_{1} \cap A_{2}$ and $\sigma_{2}=B_{1} \cap B_{2}$. Thus $h(\sigma \times \sigma)=\sigma_{1} \times \sigma_{2}$.

As $\partial(\sigma \times \sigma) \subset \partial(\sigma \times S) \cup \partial(S \times \sigma)$ we have $\partial\left(\sigma_{1} \times \sigma_{2}\right) \subset \partial\left(A_{1} \times B_{1}\right) \cup \partial\left(A_{2} \times B_{2}\right)$. Consequently, since $A_{1}$ is an arc, $B_{1}$ is a circle and $\left\{A_{2}, B_{2}\right\}$ consists of an arc and a circle, it follows that $A_{2}$ is a circle and $B_{2}$ is an arc and that $A_{1} \subset A_{2}$ and $B_{2} \subset B_{1}$. Hence $h(\sigma \times \sigma)=A_{1} \times B_{2}$. Combining the above properties we conclude that $h(\partial \sigma \times \sigma)=h(\partial \sigma \times S) \cap h(\sigma \times \sigma)=\left(\partial A_{1} \times A_{2}\right) \cap\left(A_{1} \times B_{2}\right)=\partial A_{1} \times B_{2}$ and, likewise, $h(\sigma \times \partial \sigma)=A_{1} \times \partial B_{2}$.

Theorem 8.2. Let $P$ be a $\theta$-curve with vertices $a_{0}$ and $a_{1}$ and let $\sigma$ be an edge of $P$. Let $X=(P \times P) \cup D$, where $D$ is a disc and $A=(P \times P) \cap D$ is an arc. Suppose the following conditions are fulfilled:

(i) $A \subset \partial D$;

(ii) $\left(a_{0}, a_{0}\right)$ is an endpoint of $A$;

(iii) $A \backslash\left\{\left(a_{0}, a_{0}\right)\right\}$ lies in the interior of the 2-cell $\sigma \times \sigma$.

Then $X$ does not embed in any product of two graphs, although it can be embedded in a product of two curves.

${ }^{10} \mathrm{~A} C W$-complex is said to be regular if the characteristic maps of all cells are homeomorphisms. The operation of the product " $\square$ " defined so far for cell complexes has a natural extension to regular $C W$-complexes. 
Proof. In order to prove the first assertion suppose there is an embedding $h: X \rightarrow$ $Y_{1} \times Y_{2}$ into a product of two graphs. By Lemma 8.1 there exist arcs $\sigma_{i} \subset Y_{i}$, $i=1,2$, such that $h(\sigma \times \sigma)=\sigma_{1} \times \sigma_{2}$ and $h \mid \sigma \times \sigma: \sigma \square \sigma \rightarrow \sigma_{1} \square \sigma_{2}$. Since each $Y_{j}$ is a graph, there is a neighborhood $G$ of $h\left(a_{0}, a_{0}\right)$ in $Y_{1} \times Y_{2}$ such that $G \cap\left(\stackrel{\circ}{\sigma}_{1} \times \stackrel{\circ}{\sigma}_{2}\right)$ is open in $Y_{1} \times Y_{2}$. Then there is a point $p \in A \backslash\left\{\left(a_{0}, a_{0}\right)\right\}$ such that $h(p) \in G \cap\left(\stackrel{\circ}{\sigma}_{1} \times \stackrel{\circ}{\sigma}_{2}\right)$. Hence there is a point $q \in D \backslash(P \times P)$ such that $h(q) \in G \cap\left(\stackrel{\circ}{\sigma}_{1} \times \stackrel{\circ}{\sigma}_{2}\right)$. Thus $h(q) \in h(P \times P)$, a contradiction.

Now we shall show that $X$ can be embedded in a product $Y \times Y$, where $Y$ is a curve (actually, $Y$ will be a local dendrite of order $\leq 3$ at each point and a local tree at all but one point). To construct $Y$ we proceed as follows. First, we choose a monotone sequence $a_{2}, a_{3}, \cdots$ of different points lying in the interior of $\sigma$ and converging to $a_{0}$. Then define $Y$ to be the union $Y=P \cup a_{2} b_{2} \cup a_{3} b_{3} \cup \cdots$, where $a_{2} b_{2}, a_{3} b_{3}, \cdots$ is a null-sequence of mutually disjoint arcs (converging to $a_{0}$ ) such that each $a_{n} b_{n}$ meets $P$ only at $a_{n}$. Next we define an arc $A^{\star}$ in $\sigma \times \sigma$ and a disc $D^{\star}$ in $Y \times Y$ by the formulas

$$
\begin{gathered}
A^{\star}=\left\{\left(a_{0}, a_{0}\right)\right\} \cup \bigcup_{n \geq 2}\left(\left\{a_{n}\right\} \times a_{n} a_{n+1} \cup a_{n} a_{n+1} \times\left\{a_{n+1}\right\}\right), \\
D^{\star}=\left\{\left(a_{0}, a_{0}\right)\right\} \cup \bigcup_{n \geq 2}\left[a_{n} b_{n} \times\left(a_{n} b_{n} \cup a_{n} a_{n+1} \cup a_{n+1} b_{n+1}\right) \cup a_{n} a_{n+1} \times a_{n+1} b_{n+1}\right] .
\end{gathered}
$$

Then $D^{\star} \cap(P \times P)=A^{\star}$. Now we are ready to describe the embedding.

First, define an embedding $(P \times P) \backslash(\stackrel{\circ}{\sigma} \times \stackrel{\circ}{\sigma}) \rightarrow Y \times Y$ to be the inclusion. Then extend this embedding to an embedding $P \times P \rightarrow Y \times Y$ which maps $\sigma \times \sigma$ onto itself and $A$ onto $A^{\star}$. The resulting embedding can be further extended to an embedding $X \rightarrow Y \times Y$ which maps $D$ onto $D^{\star}$. This completes the proof.

\section{A RAMIFIED 2-MANIFOLD IN A PRODUCT OF TWO GRAPHS WITH TRIVIAL 2ND COHOMOLOGY}

In an earlier paper by the authors it has been proved that if $X$ is a compactum in a product of $n$ curves with $H^{n}(X) \neq 0$, then $\operatorname{rank} H^{1}(X) \geq n$ (cf. [K-K-S]). The Structure Theorem of the present paper asserts that the same conclusion holds if the hypothesis $H^{n}(X) \neq 0$ is replaced by $X$ being a connected and locally connected quasi- $n$-manifold. One might wonder if the second hypothesis implies the first one. For this reason in the original version of this paper we posed the following problem:

Let $X$ be a connected and locally connected quasi-n-manifold in a product of $n$ graphs. Is it true that $H^{n}(X) \neq 0$ ?

Obviously, the answer is positive for $n=1$. The referee has shown that it is negative for every $n \geq 4$. Here is his presentation of the solution. (We thank the referee for permission to include his result in our paper.)

Solution for $n=4$. Let $[m]$ denote the finite set $\{1, \cdots, m\}$ and let $G_{m}$ be the graph $I \times[2] \cup \partial I \times([2] *[m])$. Then for each $m \geq 2$ there exists a quasi-2-manifold $M_{m}$ in $G_{m} \times G_{m}$ with $H^{2}\left(M_{m}\right) \simeq \mathbb{Z} / m$. Namely, $M_{m}$ is the union of the following subpolyhedra of $G_{m} \times G_{m}$ :

$$
\begin{gathered}
(I \times[2]) \times(\partial I \times([2] *[m])) \text { and }(\partial I \times([2] *[m])) \times(I \times[2]) \\
(\{1\} \times([2] *\{k\})) \times(\{1\} \times([2] *\{k+1\})) \text { and } \\
(\{i\} \times([2] *\{k\})) \times(\{j\} \times([2] *\{k\})) \text { for }(i, j)=(0,0),(0,1),(1,0)
\end{gathered}
$$


(here $m+1=1)$. Hence $H^{4}\left(M_{2} \times M_{3}\right) \simeq \mathbb{Z} / 2 \otimes \mathbb{Z} / 3=0$. By construction the quasi-4-manifold $M_{2} \times M_{3}$ embeds in a product of four graphs.

To obtain an $n$-dimensional example, for $n>4$, one takes the $n$-dimensional product $\left(M_{2} \times M_{3}\right) \times \mathbb{S}^{1} \times \cdots \times \mathbb{S}^{1}$ and inductively applies Corollary 2.3 .

It turns out that by adding a new element to the referee's idea one obtains another negative solution, for all $n \geq 2$.

Solution for $n=2$. Let $K$ be a 1 -dimensional $C W$-complex composed of four 0 -cells $u_{0}, v_{0}, u_{1}, v_{1}$ and ten 1 -cells: $A_{i}, B_{i}, C_{i}$, each joining $u_{i}$ and $v_{i}$, where $i=0,1$; $D$, joining $u_{0}$ and $u_{1} ; D^{\prime}$, joining $u_{0}$ and $v_{1} ; E$, joining $v_{0}$ and $v_{1} ; E^{\prime}$, joining $v_{0}$ and $u_{1}$. Put $T_{i}=A_{i} \cup B_{i} \cup C_{i}$ and let $M$ denote the union of the following subpolyhedra of $|K| \times|K|$ :

$$
\begin{gathered}
\left(T_{0} \times\left(D^{\prime} \cup E^{\prime}\right)\right) \cup\left(T_{1} \times(D \cup E)\right) \cup\left((D \cup E) \times\left(T_{0} \cup T_{1}\right)\right), \\
\left(A_{1} \times B_{1}\right) \cup\left(B_{1} \times C_{1}\right) \cup\left(C_{1} \times A_{1}\right) \text { and } \\
\left(A_{i} \times A_{j}\right) \cup\left(B_{i} \times B_{j}\right) \cup\left(C_{i} \times C_{j}\right) \text { for }(i, j)=(0,0),(0,1),(1,0) .
\end{gathered}
$$

Let $L$ denote the subcomplex of $K \square K$ such that $M=|L|$. Notice that $M$ is a union of 2-cells of $L$ and that each 1-cell in $L$ is a face of either two or three 2-cells of $L$. Hence $M$ is a ramified 2-manifold. One can show that for any two 2-cells $\sigma, \sigma^{\prime} \in L$ there exists a sequence $\sigma_{1}, \cdots, \sigma_{n}$ of 2-cells in $L$ with $\sigma_{1}=\sigma$ and $\sigma_{n}=\sigma^{\prime}$ such that

(a) the intersection of any two consecutive 2-cells $\sigma_{i}$ and $\sigma_{i+1}$ is a 1-cell of $L$ that is not a face of any other 2-cell of $L$, and

(b) if $\sigma=\sigma^{\prime}$ and each $\sigma_{i}$ is assigned an orientation so that any two consecutive 2 -cells are coherently oriented, then the orientations of $\sigma_{1}$ and $\sigma_{n}$ give opposite orientations on $\sigma$.

Since in addition there is a 1-cell in $L$ which is a face of three 2-cells of $L$, we conclude that $H^{2}(M)=0$.

By the same argument as above we obtain the main result of this section.

Theorem 9.1. For each $n \geq 2$ there is a polyhedron $X$ lying in a product of $n$ graphs that is a quasi-n-manifold with $H^{n}(X)=0$.

\section{APPENDIX}

The notion of a quasi- $n$-manifold was created with the purpose of proving a general theorem (exemplified here by the Factorization Theorem) which in particular would resolve a problem of $\mathrm{R}$. Cauty. Until a report from the referee we were quite satisfied with that notion. However, the referee suggested two other classes of $n$ dimensional compacta defined in terms of algebraic topology: $h$-n-manifolds and $c$-n-manifolds. For the new classes, as observed by the referee, some basic results of our paper formulated originally for quasi- $n$-manifolds were also true. Prompted by the referee's suggestion, we have defined a more general and geometric class of compacta possessing the basic properties of quasi- $n$-manifolds. That class has been named weak $n$-manifolds. The proofs of the results extended to weak $n$-manifolds are essentially the same as the original proofs for quasi- $n$-manifolds.

We have already observed that quasi- $n$-manifolds are included in weak $n$-manifolds. One can show that the same is true for both h- $n$-manifolds and c- $n$-manifolds. Below we prove this only for h- $n$-manifolds. 
An $n$-dimensional compactum $X$ is said to be an $h$-n-manifold at a point $x \in X$ if $H_{n}(X, X \backslash\{x\}) \neq 0$. By definition, $H_{i}(X, X \backslash\{x\})=\lim _{\rightarrow} H_{i}(X, X \backslash U)$, where $U$ runs over all open neighborhoods of $x$ in $X$. Here $H_{*}$ is the Steenrod homology with integer coefficients defined on compact pairs [St; cf. [M], Mil, $\underline{\underline{S}}$. The compactum $X$ is said to be an $h$-n-manifold if $X$ is an h- $n$-manifold at every point $x \in X$.

Theorem. If $X$ is an $\mathrm{h}-n$-manifold at a point $x \in X$, then $X$ is a weak $n$-manifold at $x$.

In order to prove this theorem we need the following

Lemma. If $B$ is a closed subset of an m-dimensional contractible compactum $Y$, then $\widetilde{H}_{m}(B)=0$ for each $m \geq 0$.

Proof. We can assume that $B \neq \emptyset$. Then $H_{m+1}(Y, B)=0$ because $H_{m+1}(Y, B) \simeq$ $H_{m+1}(Y / B,[B]) \simeq H_{m+1}(Y / B)$ and the latter group is trivial as $Y / B$ is a compactum with $\operatorname{dim} Y / B<m+1$. Since $Y$ is contractible we have $\widetilde{H}_{m}(Y)=0$. The conclusion follows from the exactness of the reduced homology sequence of $(Y, B)$ :

$$
\cdots \leftarrow \widetilde{H}_{m}(Y) \leftarrow \widetilde{H}_{m}(B) \leftarrow H_{m+1}(Y, B) \leftarrow \cdots .
$$

Proof of the Theorem. By the hypothesis there is an open set $U_{0} \ni x$ and an element $a \in H_{n}\left(X, X \backslash U_{0}\right)$ representing a non-zero element of $\lim _{\rightarrow} H_{n}(X, X \backslash U)$. Suppose $X$ is not a weak $n$-manifold at $x$. Then there is an open set $U \ni x$ with $\bar{U} \subset U_{0}$ such that the compact pair $(\bar{U}, \partial U)$ extends to a compact pair $(P, Q)$ of absolute retracts with $\operatorname{dim}(P, Q)=(n, n-1)$. Setting $(Y, B)=(P, Q)$ and

applying the Lemma we infer that $\widetilde{H}_{n-1}(\partial U)=0$ and $H_{n}(\bar{U})=0$. Since the sequence $\widetilde{H}_{n-1}(\partial U) \leftarrow H_{n}(\bar{U}, \partial U) \leftarrow H_{n}(\bar{U})$ is exact, we have $H_{n}(\bar{U}, \partial U)=0$. Since the inclusion map $(\bar{U}, \partial U) \rightarrow(X, X \backslash U)$ is a relative homeomorphism we have $H_{n}(\bar{U}, \partial U) \simeq H_{n}(X, X \backslash U)$. Hence $H_{n}(X, X \backslash U)=0$. It follows that the restriction $\left.a\right|_{U} \in H_{n}(X, X \backslash U)$ is the zero element, a contradiction.

\section{PROBLEMS}

Problem 1. Characterize 2-dimensional polyhedra which can be embedded in a product of two graphs.

Problem 2. Characterize closed 3-manifolds which can be embedded in a product of three graphs.

Problem 3. Let $K$ be a 2-dimensional simplicial complex that collapses on a subcomplex $L$. Suppose $|L|$ embeds in a product of two curves.

(a) Does every embedding of $|L|$ in the product $\mu \times \mu$ (where $\mu$ is the Menger curve) extend to an embedding of $|K|$ ?

(b) Does $K$ embed in a product of two curves?

Problem 4. Let $X$ be a polyhedron that is a weak $n$-manifold, $n \geq 2$, and let $\left(f_{1}, \cdots, f_{n}\right): X \rightarrow Y_{1} \times \cdots \times Y_{n}$ be an embedding in a product of curves. Is it possible to approximate mappings $f_{i}$ by mappings $f_{i}^{\prime}: X \rightarrow Y_{i}$ so that $\left(f_{1}^{\prime}, \cdots, f_{n}^{\prime}\right)$ is still an embedding and each $f_{i}^{\prime}(X)$ is a graph? 
Problem 5. Let $X_{i}, i=1,2$, be a weak $n_{i}$-manifold at a point $x_{i}$. Is their product $X_{1} \times X_{2}$ a weak $\left(n_{1}+n_{2}\right)$-manifold at $\left(x_{1}, x_{2}\right)$ ? In particular, is $X_{1} \times X_{2}$ a weak $\left(n_{1}+1\right)$-manifold at $\left(x_{1}, x_{2}\right)$ if $X_{1}$ is a weak $n_{1}$-manifold at $x_{1}$ and $X_{2}$ is a 1 -manifold at $x_{2}$ ?

Problem 6. Let $X_{1}$ be a quasi- $n_{1}$-manifold at $x_{1} \in X_{1}$ and let $X_{2}$ be a 1-manifold at $x_{2} \in X_{2}$. Is $X_{1} \times X_{2}$ a quasi- $\left(n_{1}+1\right)$-manifold at $\left(x_{1}, x_{2}\right)$ ?

Remark. Recall that if both $X_{i}, i=1,2$, are polyhedral quasi- $n_{i}$-manifolds at $x_{i} \in X_{i}$ and $n_{2} \leq 2$, then $X_{1} \times X_{2}$ is a quasi- $\left(n_{1}+n_{2}\right)$-manifold at $\left(x_{1}, x_{2}\right)$ (see Corollary 2.3) 11

\section{ACKNOWLEDGMENTS}

The authors express kind thanks to the referee for his comment on the original version of this article. They are indebted for numerous remarks and suggestions which have led to a serious modification of the presentation. The referee's solution, for $n \geq 4$, of the problem from Section 9 inspired the final solution (for $n \geq 2$ ). The characterization of polyhedral quasi-manifolds in terms of local cohomologies presented in Section 4 is essentially due to him. Proposition 2.7 strengthens another observation of the referee. The authors also thank him for pointing out reference G-M-R.

\section{REFERENCES}

[B1] K. Borsuk, Über das Phänomen der Unzerlegbarkeit in der Polyedertopologie, Comment. Math. Helv. 8 (1935), 142-148. MR1509522

[B2] - Theory of Retracts, PWN-Polish Scientific Publishers, Warszawa, 1967.

[B3] , Remarks on the Cartesian product of two 1-dimensional spaces, Bull. Polish Acad. Sci. Math. 23 (1975), 971-973. MR0394636 (52:15437)

[Bo] H. G. Bothe, Eine Einbettung $m$-dimensionaler Mengen in einen $(m+1)$-dimensionalen absoluten Retrakt, Fund. Math. 52 (1963), 209-224. MR0151972 (27:1953)

[Bw] B.W. Bowers, General position properties satisfied by finite products of dendrites, Trans. Amer. Math. Soc. 288 (1985), 739-753. MR776401 (86f:54063)

[C] R. Cauty, Sur le plongement des surfaces non orientables dans un produit de deux graphes, Bull. Polish Acad. Sci. Math. 32 (1984), 121-128. MR766989 (86c:57015)

[E-S] S. Eilenberg and N. Steenrod, Foundations of Algebraic Topology, Princeton University Press, Princeton, New York, 1952. MR0050886 (14:398b)

[E] R. Engelking, Dimension Theory, PWN-Polish Scientific Publishers, Warszawa; NorthHolland Publishing Company, Amsterdam, Oxford, New York, 1978. MR0482697 (58:2753b)

[G-M-R] D. Gillman, S. Matveev and D. Rolfsen, Collapsing and reconstruction of manifolds, Contemp. Math. 164 (1994), 35-39. MR1282753 (95c:57036)

[H] A. Hatcher, Algebraic Topology, Cambridge University Press, 2002. MR.1867354 (2002k:55001)

[H-W] W. Hurewicz and H. Wallman, Dimension Theory, Princeton, 1948.

[I-M] I. Ivanšić and U. Milutinović, A universal separable metric space based on the triangular Sierpiński curve, Top. Appl. 120 (2002), 237-271. MR.1895494(2003a:54039)

$[\mathrm{K}] \quad$ Y. Kodama, On embeddings of spaces into ANR and shapes, J. Math. Soc. Japan 27 (1975), 533-544. MR0400158 (53:3993)

[K-O] Y. Kodama and J. Ono, On two notions of shape for pairs of spaces, General Topology and Appl. 6 (1976), 207-225. MR0394554(52:15355)

\footnotetext{
${ }^{11}$ The dimensional restriction $n_{2} \leq 2$ is essential. In fact, setting $X_{1}=\sum M_{2}$ and $X_{2}=\sum M_{3}$, where $M_{2}$ and $M_{3}$ are the polyhedra defined in Section 9 , one gets two quasi-3-manifolds such that $X_{1} \times X_{2}$ is not a quasi-6-manifold.
} 
[K-K-S] A. Koyama, J. Krasinkiewicz, S. Spiez, Embedding compacta into products of curves, arXiv:0712.3470v1 [math.GT] 20 Dec 2007, 1-71.

[K-K-S 1] _ On embeddings into products of curves - An algebraic approach, preprint.

[Kr1] J. Krasinkiewicz, On approximation of mappings into 1-manifolds, Bull. Polish Acad. Sci. Math. 44 (1996), 431-440. MR1420956 (97k:54011)

[Kr2] On a method of constructing ANR-sets. An application of inverse limits, Fund. Math. 92 (1976), 95-112. MR.0420546 (54:8560)

[Ku] W. Kuperberg, On embeddings of manifolds into Cartesian products of compacta, Bull. Acad. Pol. Sci. Ser. Math. 26 (1978), 845-848. MR518991 (81i:57011)

[Kur] K. Kuratowski, Topology, vol. II, PWN-Academic Press, Warsaw, New York, 1968. MR.0259835 (41:4467)

[L] S.L. Lipscomb, On embedding finite-dimensional metric spaces, Trans. Amer. Math. Soc. 211 (1975), 143-160. MR0380751 (52:1648)

[M] W. S. Massey, Homology and Cohomology Theory, Marcel Dekker, New York, 1978; the author's guide for reading this book How to give an exposition of the Čech-AlexanderSpanier type homology theory, Amer. Math. Monthly 85 (1978), 75-83. MR0488016 (58:7594), MR0488017(58:7595)

[Ma] J. P. May, A Concise Course in Algebraic Topology, The University of Chicago Press, Chicago and London, 1999. MR1702278(2000h:55002)

[Mi] D. Michalik, Embeddings of $n$-dimensional separable metric spaces into the product of Sierpiński curves, Proc. Amer. Math. Soc. 138(8) (2007), 2661-2664. MR2302589 (2007m:54031)

[Mil] J. Milnor, On the Steenrod homology theory, Novikov Conjectures, Index Theorems and Rigidity (vol. 1), London Math. Soc. Lecture Note Ser., vol. 226, Cambridge Univ. Press, 1995, pp. 79-96. MR.1388297 (98d:55005)

[N1] J. Nagata, Note on dimension theory for metric spaces, Fund. Math. 45 (1958), 143181. MR0105081 (21:3827)

[N2] _ Modern Dimension Theory, North-Holland, Amsterdam, 1965.

[Ol] W. Olszewski, Embeddings of finite-dimensional spaces into finite product of 1dimensional spaces, Top. Appl. 40 (1985), 93-99. MR.1114094 (92g:54051)

[P] R. Pol, A 2-dimensional compactum in the product of two 1-dimensional compacta which does not contain any rectangle, Topology Proc. 16 (1991), 133-135. MR1206460 (94b:54101)

[R-S] C. P. Rourke and B. J. Sanderson, Introduction to Piecewise-Linear Topology, SpringerVerlag, Berlin, Heilderberg, New York, 1982. MR665919 (83g:57009)

[S] E. G. Sklyarenko, Homology and cohomology of general spaces, General Topology II. Encycl. Math. Sci., vol. 50, 1996, pp. 119-246, English transl. MR.1392482

[Sp] E. Spanier, Algebraic Topology, McGraw-Hill, New York, 1966. MR0210112 (35:1007)

[St] N. E. Steenrod, Regular cycles of compact metric spaces, Ann. Math. 41 (1940), 833851. MR0002544 (2:73c)

[Ste] Y. Sternfeld, Mappings in dendrites and dimension, Houston J. Math. 19 (1993), 483497. MR 1242434 (94j:54017)

[T] K. Tsuda, A note on closed embeddings of finite dimensional metric spaces II, Bull. Pol. Acad. Sci. 33 (1985), 541-546. MR826381 (87g:54070b)

[W] G. T. Whyburn, Analytic Topology, vol. 28, Amer. Math. Soc. Colloquium Publications, 1942. MR0007095 (4:86b)

[Z] E. C. Zeeman, On the dunce hat, Topology 2 (1964), 341-358. MR0156351 (27:6275)

Department of Mathematics, Faculty of Science, Shizuoka University, Suruga, SHIZUOKA, 422-8529, JAPAN

E-mail address: sakoyam@ipc.shizuoka.ac.jp

The Institute of Mathematics, Polish Academy of Sciences, Ul. Śniadeckich 8, 00-956, WARSAW, POLAND

E-mail address: jokra@impan.pl

The Institute of Mathematics, Polish Academy of Sciences, Ul. Śniadeckich 8, 00-956, WarsaW, Poland

E-mail address: spiez@impan.pl 